\title{
An Unified Parametric-Nonparametric Uncertainty Quantification Approach for Linear Dynamical Systems
}

\author{
Sondipon Adhikari ${ }^{*}$ \\ University of Wales Swansea. Swansea, United Kingdom
}

\begin{abstract}
Uncertainties need to be taken into account for credible predictions of the dynamic response of complex aerospace structural systems. Such uncertainties include uncertainties in the system parameters (parametric uncertainty) and those arising due to the modelling of a complex system (nonparametric uncertainty). Over the past four decades distinct methods have been developed to quantify these two types of uncertainties seperately. In this paper we proposed an unified approach to model both parametric and nonparametric uncertainties simultaneously. The proposed approach is based on a noncentral Wishart distribution whose parameters are obtained such that the covariance matrices of the elements of the mass, stiffness and damping matrices arising due to parametric uncertainties are satisfied in a least-square sense. The existing nonparametric distribution (a central Wishart distribution) can be obtained as a special case of the proposed noncentral Wishart distribution. A simple simulation algorithm is developed to implement the noncentral Wishart random matrix model in conjunction with the conventional finite element method. The method is applied to the vibration of a cantilever plate with uncertainties. The dynamic response obtained using the noncentral Wishart random matrix model agrees well with the results obtained from the stochastic finite element method.
\end{abstract}

\section{Nomenclature}

$\overline{\mathbf{G}}$

$\mathbf{C}_{G}$

$\mathbf{D}(\omega)$

$\mathbf{f}(t)$

$\mathbf{G}$

$\mathbf{G}_{n}$

$\mathbf{H}(\omega)$

$\mathbf{H}_{n}$

$\mathbf{I}_{n}$

$\mathbf{K}_{n n}$

$\mathbf{M}, \mathbf{C}$ and $\mathbf{K}$

$\mathbf{O}_{n, m}$

$\boldsymbol{\Omega}, \boldsymbol{\Theta}$

$\mathbf{q}(t)$

$\Gamma_{n}(a)$

$\nu$

$\omega$

$\phi(\bullet)$

$\operatorname{vech}(\bullet)$

$\operatorname{vec}(\bullet)$

$n$

$p, \boldsymbol{\Sigma}$ mean of a system matrix

$n^{2} \times n^{2}$ covariance matrix of a system matrix

dynamic stiffness matrix

forcing vector

symbol for a system matrix, $\mathbf{G} \equiv\{\mathbf{M}, \mathbf{C}, \mathbf{K}\}$

$n^{2} \times n(n+1) / 2$-dimensional duplication matrix

frequency response function (FRF) matrix

Left Moore-Penrose inverse of the duplication matrix $\mathbf{G}_{n}$ identity matrix of dimension $n$

$n^{2}$ dimensional commutation matrix ${ }^{1}$ (also known as the vec-permutation matrix ${ }^{2}$ )

mass, damping and stiffness matrices respectively

null matrix of dimension $n \times m$

a $n \times n$ real matrix parameters of noncentral Wishart distribution

response vector

multivariate gamma function

order of the inverse-moment constraint

excitation frequency

characteristic function of of $(\bullet)$

vech operation on a symmetric matrix

vec operation on a matrix

number of degrees of freedom

scalar and matrix parameters of the Wishart distribution

${ }^{*}$ Chair of Aerospace Engineering School of Engineering University of Wales Swansea Singleton Park, Swansea SA2 8PP, UK, AIAA Senior Member.

Copyright (c) 2007 by the American Institute of Aeronautics and Astronautics, Inc. The U.S. Government has a royalty-free license to exercise all rights under the copyright claimed herein for Governmental purposes. All other rights are reserved by the copyright owner. 


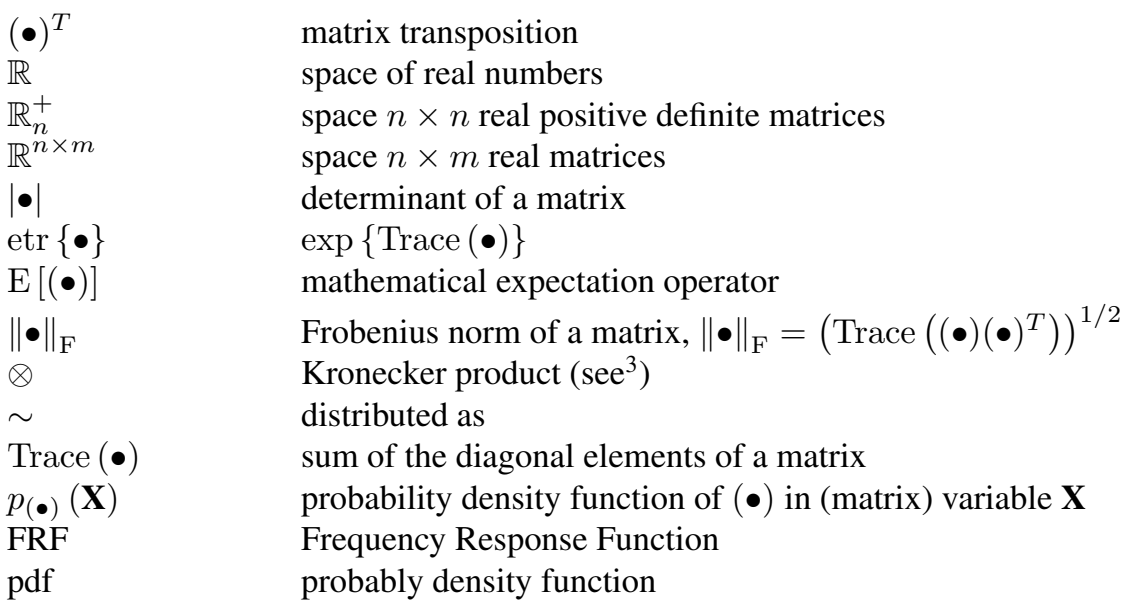

\section{Introduction}

$\mathrm{U}$ NCERTAINTIES are unavoidable in the description of complex aerospace structures. The quantification of uncertainties plays a crucial role in establishing the credibility of a numerical model. ${ }^{4,5}$ Uncertainties can be broadly divided into two categories. The first type is due to the inherent variability in the system parameters, for example, different cars manufactured from a single production line are not exactly the same. This type of uncertainty is often referred to as aleatoric uncertainty and generally considered to be irreducible. If enough samples are present, it is possible to characterize the variability using the well established statistical methods and consequently the probably density functions (pdf) of the parameters can be obtained. The second type of uncertainty is due to the lack of knowledge regarding a system, often referred to as epistemic uncertainty. This kind of uncertainty generally arise in the modelling of complex systems, for example, in the modeling of cabin noise in helicopters. Due to its very nature, it is comparatively difficult to quantify or model this type of uncertainties. This uncertainty is reducible provided more knowledge regarding the system is available. There are two broad approaches to quantify uncertainties in a model. The first is the parametric approach and the second is the non-parametric approach. In the parametric approach the uncertainties associated with the system parameters, such as Young's modulus, mass density, Poisson's ratio, damping coefficient and geometric parameters are quantified using statistical methods and propagated, for example, using the stochastic finite element method. ${ }^{6-15}$ This type of approach is suitable to quantify aleatoric uncertainties. Epistemic uncertainty on the other hand do not explicitly depend on the system parameters. For example, there can be unquantified errors associated with the equation of motion (linear on non-linear), in the damping model (viscous or non-viscous ${ }^{16}$ ), in the model of structural joints, and also in the numerical methods (e.g, discretisation of displacement fields, truncation and roundoff errors, tolerances in the optimization and iterative algorithms, step-sizes in the time-integration methods). It is evident that the parametric approach is not suitable to quantify this type of uncertainties. As a result non-parametric approaches ${ }^{16-19}$ have been proposed for this purpose.

Although we have mentioned and made differences between the two different types of uncertainties, in practical problems it is in general very difficult, if not impossible, to distinguish them. Recently reported experimental studies by Adhikari et. al..$^{20,21}$ on one hundred nominally identical beams and plates emphasize this fact. As result, to come up with a credible numerical model of complex dynamical systems, we need to quantify and model both types of uncertainties simultaneously. Uncertainties associated with a variable can be characterized using the probabilistic approach or possibilistic approaches based on interval algebra, convex sets, Fuzzy sets or generalized DempsterSchafer theory. In this paper probabilistic approach has been adopted. The equation of motion of a damped $n$-degreeof-freedom linear dynamic system can be expressed as

$$
\mathbf{M} \ddot{\mathbf{q}}(t)+\mathbf{C} \dot{\mathbf{q}}(t)+\mathbf{K q}(t)=\mathbf{f}(t)
$$

where $\mathbf{M}, \mathbf{C}$ and $\mathbf{K}$ are the mass, damping and stiffness matrices respectively. In order to completely quantify the uncertainties associated with system (1) we need to obtain the probability density functions of the random matrices $\mathbf{M}$, $\mathbf{C}$ and $\mathbf{K}$. Using the parametric approach, such as the stochastic finite element method, one usually obtains a problem specific covariance structure for the elements of system matrices. The nonparametric approach ${ }^{17-19}$ on the other hand results in a central Wishart distribution for the system matrices. The central Wishart matrix has its own covariance 
structure which in general may not agree with the covariance structure obtained using the parametric method. This is one of the main difficulty in using the central Wishart matrices for parametric uncertainty quantification.

The aim of this paper is to develop a parametric-nonparametric uncertainty quantification tool which can simultaneously model both types of uncertainties in an unified manner. The overall uncertainly modeling approach is diagrammatically explained in Fig. 1. The approach proposed here is based on noncentral Wishart distribution. The noncentral Wishart distribution has more parameters compared to the central Wishart distribution. As a result it is more capable to capture the parametric nature of uncertainty. The central idea of the paper is to exploit this additional statistical degrees of freedom provided by the noncentral distribution. A least-square error minimization approach is developed to obtain the parameters of the noncentral distribution so that it satisfy the covariance matrix of each of the system matrices appearing in Eq. (1). A simple and easy-to-implement simulation algorithm is given to implement the new approach. In the next section we briefly outline some aspects of random matrix theory required for further

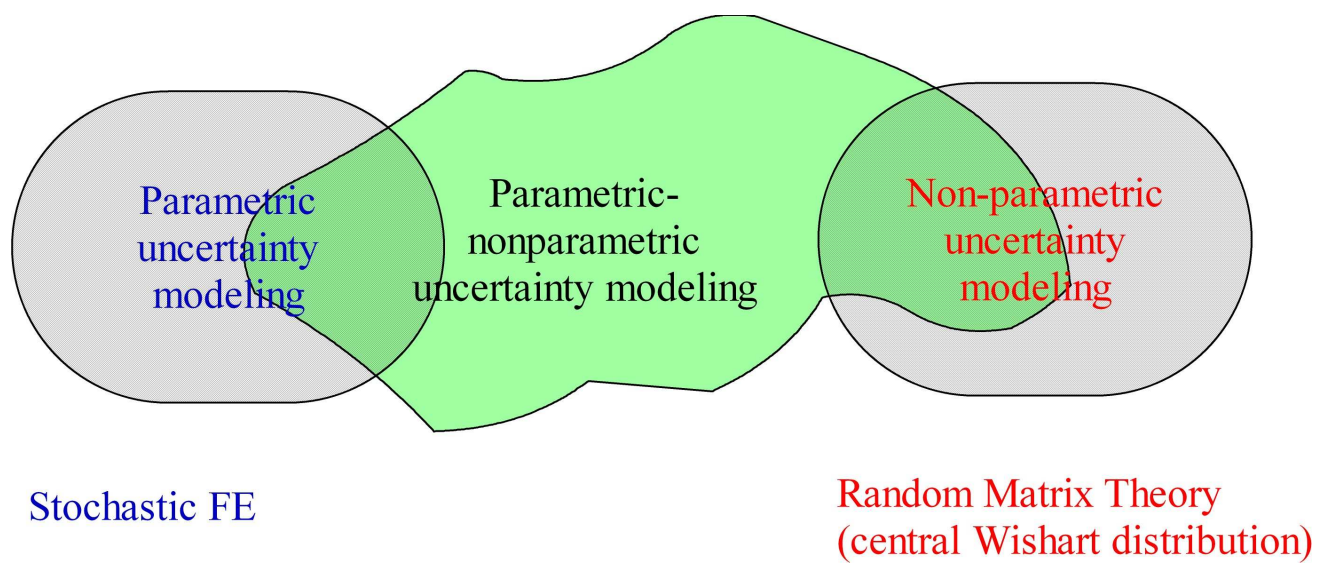

Figure 1. Symbolic representation of the proposed parametric-nonparametric uncertainly modeling in structural dynamics.

developments.

\section{Brief Review of Random Matrix Distributions}

In this section we introduce the concept of matrix variate probability density functions or random matrices. Random matrices were introduced by Wishart ${ }^{22}$ in the late 1920 s in the context of multivariate statistics. Research on random matrices has attracted interests in multivariate statistics, physics, number theory and more recently in mechanical and electrical engineering. We refer the books ${ }^{23-28}$ for the history and applications of random matrix theory. The probability density function of a random matrix can be defined in a manner similar to that of a random variable or random vector. If $\mathbf{A}$ is a $n \times m$ real random matrix, then the matrix variate probability density function of $\mathbf{A} \in \mathbb{R}^{n \times m}$, denoted by $p_{\mathbf{A}}(\mathbf{A})$, is a mapping from the space of $n \times m$ real matrices to the real line, i.e., $p_{\mathbf{A}}(\mathbf{A}): \mathbb{R}^{n \times m} \rightarrow \mathbb{R}$. Here we define probability density functions of few random matrices which are relevant to stochastic mechanics problems.

Gaussian random matrix: A rectangular random matrix $\mathbf{X} \in \mathbb{R}^{n \times p}$ is said to have a matrix variate Gaussian distribution with mean matrix $\mathcal{M} \in \mathbb{R}^{n \times p}$ and covariance matrix $\boldsymbol{\Sigma} \otimes \boldsymbol{\Psi}$, where $\boldsymbol{\Sigma} \in \mathbb{R}_{n}^{+}$and $\boldsymbol{\Psi} \in \mathbb{R}_{p}^{+}$provided the pdf of $\mathbf{X}$ is given by

$$
p_{\mathbf{X}}(\mathbf{X})=(2 \pi)^{-n p / 2}|\boldsymbol{\Sigma}|^{-p / 2}|\boldsymbol{\Psi}|^{-n / 2} \operatorname{etr}\left\{-\frac{1}{2} \boldsymbol{\Sigma}^{-1}(\mathbf{X}-\mathcal{M}) \Psi^{-1}(\mathbf{X}-\mathcal{M})^{T}\right\} .
$$

This distribution is usually denoted as $\mathbf{X} \sim N_{n, p}(\mathcal{M}, \boldsymbol{\Sigma} \otimes \boldsymbol{\Psi})$.

Wishart matrix: A $n \times n$ symmetric positive definite random matrix $\mathbf{S}$ is said to have a Wishart distribution with parameters $p \geq n$ and $\Sigma \in \mathbb{R}_{n}^{+}$, if its pdf is given by

$$
p_{\mathbf{S}}(\mathbf{S})=\left\{2^{\frac{1}{2} n p} \Gamma_{n}\left(\frac{1}{2} p\right)|\boldsymbol{\Sigma}|^{\frac{1}{2} p}\right\}^{-1}|\mathbf{S}|^{\frac{1}{2}(p-n-1)} \operatorname{etr}\left\{-\frac{1}{2} \boldsymbol{\Sigma}^{-1} \mathbf{S}\right\} .
$$

This distribution is usually denoted as $\mathbf{S} \sim W_{n}(p, \mathbf{\Sigma})$. Using a maximum entropy approach, Adhikari ${ }^{18,19}$ proved that the system matrices arising in linear structural dynamics should be Wishart matrices. 
Matrix variate gamma distribution: A $n \times n$ symmetric positive definite random matrix $\mathbf{W}$ is said to have a matrix variate gamma distribution with parameters $a$ and $\Psi \in \mathbb{R}_{n}^{+}$, if its pdf is given by

$$
p_{\mathbf{W}}(\mathbf{W})=\left\{\Gamma_{n}(a)|\boldsymbol{\Psi}|^{-a}\right\}^{-1}|\mathbf{W}|^{a-\frac{1}{2}(n+1)} \operatorname{etr}\{-\mathbf{\Psi} \mathbf{W}\} ; \quad \Re(a)>\frac{1}{2}(n-1) .
$$

This distribution is usually denoted as $\mathbf{W} \sim G_{n}(a, \mathbf{\Psi})$. The matrix variate gamma distribution has been used by Soize $^{17}$ for the random system matrices of linear dynamical systems.

The main difference between the matrix variate gamma distribution and the Wishart distribution is that historically only integer values were considered for the shape parameter $p$ in the Wishart matrices. It is however a misconception that the scope of Wishart distribution is limited to the integer values of $p$ only (see the second para in pp. 87 in Muirhead ${ }^{27}$ and the para after Eq. (3.2.2) in Gupta and Nagar, ${ }^{29}$ pp. 89). From an analytical point of view the gamma and the Wishart distributions are identical as the gamma distribution can be related to the Wishart distribution as $W_{n}(p, \boldsymbol{\Sigma})=G_{n}\left(p / 2, \frac{1}{2} \boldsymbol{\Sigma}^{-1}\right)$. For this reason, modern random matrix theory normally does not make any distinctions between them. Because the Wishart random matrix is the oldest ${ }^{22}$ and perhaps the most widely used random matrix model, ${ }^{24,25,27}$ in this paper we present our results in terms of the Wishart matrices only.

Noncentral Wishart matrix: A $n \times n$ symmetric positive definite random matrix $\mathbf{S}$ is said to have a noncentral Wishart distribution with parameters $p \geq n, \boldsymbol{\Sigma} \in \mathbb{R}_{n}^{+}$and $\Theta \in \mathbb{R}_{n}^{+}$, if its pdf is given by

$$
p_{\mathbf{S}}(\mathbf{S})=\left\{2^{\frac{1}{2} n p} \Gamma_{n}\left(\frac{1}{2} p\right)|\boldsymbol{\Sigma}|^{\frac{1}{2} p}\right\}^{-1} \operatorname{etr}\left\{-\frac{1}{2} \boldsymbol{\Theta}\right\} \operatorname{etr}\left\{-\frac{1}{2} \boldsymbol{\Sigma}^{-1} \mathbf{S}\right\}|\mathbf{S}|^{\frac{1}{2}(p-n-1)}{ }_{0} F_{1}\left(p / 2, \boldsymbol{\Theta} \boldsymbol{\Sigma}^{-1} \mathbf{S} / 4\right) \text {. }
$$

where ${ }_{0} F_{1}$ the hypergeometric function (Bessel function) of a matrix argument. ${ }^{27,29}$ This distribution is usually denoted as $\mathbf{S} \sim W_{n}(p, \boldsymbol{\Sigma}, \boldsymbol{\Theta})$. Note that if the noncentrality parameter $\boldsymbol{\Theta}$ is a null matrix, then noncentral Wishart distribution in Eq. (5) reduces to the Wishart distribution in Eq. (3).

In Eqs. (3) $-(5)$, the function $\Gamma_{n}(a)$ is the multivariate gamma function, which can be expressed in terms of products of the univariate gamma functions as

$$
\Gamma_{n}(a)=\pi^{\frac{1}{4} n(n-1)} \prod_{k=1}^{n} \Gamma\left[a-\frac{1}{2}(k-1)\right] ; \quad \text { for } \quad \Re(a)>\frac{1}{2}(n-1) .
$$

For more details on the matrix variate distributions we refer to the books by Mezzadri and Snaith, ${ }^{23}$ Tulino and Verdú, ${ }^{24}$ Eaton, ${ }^{25}$ Girko, ${ }^{26}$ Muirhead ${ }^{27}$ and Mehta. ${ }^{28}$ Among the four types of random matrices introduced above, the distributions given by Eqs. (3) - (5) will always result in symmetric and positive definite matrices. Therefore, they can be possible candidates for modeling random system matrices arising in probabilistic structural mechanics.

\section{Limitations of the Nonparametric Distribution}

Suppose that the mean values of $\mathbf{M}, \mathbf{C}$ and $\mathbf{K}$ are given by $\overline{\mathbf{M}}, \overline{\mathbf{C}}$ and $\overline{\mathbf{K}}$ respectively. This information is likely to be available, for example, using the deterministic finite element method. However, there are uncertainties associated with our modelling so that $\mathbf{M}, \mathbf{C}$ and $\mathbf{K}$ are actually random matrices. The distribution of these random matrices should be such that they are

(a) symmetric

(b) positive-definite, and

(c) the moments of the inverse of the dynamic stiffness matrix

$$
\mathbf{D}(\omega)=-\omega^{2} \mathbf{M}+i \omega \mathbf{C}+\mathbf{K}
$$

should exist $\forall \omega$. That is, if $\mathbf{H}(\omega)$ is the frequency response function (FRF) matrix

$$
\mathbf{H}(\omega)=\mathbf{D}^{-1}(\omega)=\left[-\omega^{2} \mathbf{M}+i \omega \mathbf{C}+\mathbf{K}\right]^{-1}
$$

then the following condition must be satisfied for positive values of $\nu$ :

$$
\mathrm{E}\left[\|\mathbf{H}(\omega)\|_{\mathrm{F}}^{\nu}\right]<\infty, \quad \forall \omega
$$

$$
4 \text { of } 22
$$


Because the matrices $\mathbf{M}, \mathbf{C}$ and $\mathbf{K}$ have similar probabilistic characteristics, for notational convenience we will use the notation $\mathbf{G}$ which stands for any one the system matrices. Soize (see ${ }^{17}$ and references therein) fitted a central Wishart distribution using the entropy optimization approach and showed that $\mathbf{G} \sim W_{n}(p, \boldsymbol{\Sigma})$, where the distribution parameters are given by

$$
\begin{aligned}
p & =n+1+\theta \\
\text { and } \quad \boldsymbol{\Sigma} & =\overline{\mathbf{G}} /(n+1+\theta) .
\end{aligned}
$$

The constant $\theta$, which controls the 'randomness' of the model, can be related to the standard deviation of the random matrix as

$$
\theta=\frac{1}{\delta_{G}^{2}}\left\{1+\frac{\{\operatorname{Trace}(\overline{\mathbf{G}})\}^{2}}{\operatorname{Trace}\left(\overline{\mathbf{G}}^{2}\right)}\right\}-(n+1) .
$$

Here $\delta_{G}$ is known as the dispersion parameter which characterize the uncertainty in the random matrix G. The parameter $\delta_{G}$ is defined as

$$
\delta_{G}^{2}=\frac{\mathrm{E}\left[\|\mathbf{G}-\mathrm{E}[\mathbf{G}]\|_{\mathrm{F}}^{2}\right]}{\|\mathrm{E}[\mathbf{G}]\|_{\mathrm{F}}^{2}}=\frac{\operatorname{Trace}(\operatorname{cov}(\operatorname{vec}(\mathbf{G})))}{\operatorname{Trace}\left(\overline{\mathbf{G}}^{2}\right)} .
$$

From this expression observe that $\delta_{G}$ can be viewed as the mean-normalized standard deviation of the random matrix G. Although the distribution proposed by Soize ${ }^{17}$ produces positive definite and invertible random matrix ensemble, it was later shown ${ }^{18,19}$ that the 'mean of the inverse' and the 'inverse of the mean' can be significantly different. For large systems, when $n$ is more than several thousands, the difference between $\mathrm{E}\left[\mathbf{G}^{-1}\right]$ and $\overline{\mathbf{G}}^{-1}$ can be more than few orders of magnitude. To avoid this non-physical case, using a matrix factorization approach in conjunction with least-square error minimization, Adhikari ${ }^{18,19}$ showed that the optimal distribution of $\mathbf{G}$ should be $\mathbf{G} \sim W_{n}(p, \boldsymbol{\Sigma})$, where

$$
\begin{aligned}
p & =n+1+\theta \\
\text { and } \quad \boldsymbol{\Sigma} & =\overline{\mathbf{G}} / \alpha .
\end{aligned}
$$

The constant $\theta$ is given by Eq. (12) and $\alpha$ is given by

$$
\alpha=\sqrt{\theta(n+1+\theta)}=\sqrt{p(p-n-1)} .
$$

For these parameter selections the mean of the matrix and its inverse produce minimum deviations from their respective deterministic values.

The first moment (mean) and the elements of the covariance tensor are given by ${ }^{29}$

$$
\begin{aligned}
& \mathrm{E}[\mathbf{G}]=p \boldsymbol{\Sigma}=p \overline{\mathbf{G}} / \alpha \\
& \operatorname{cov}\left(G_{i j}, G_{k l}\right)=p\left(\Sigma_{i k} \Sigma_{j l}+\Sigma_{i l} \Sigma_{j k}\right)=\frac{1}{\theta}\left(\bar{G}_{i k} \bar{G}_{j l}+\bar{G}_{i l} \bar{G}_{j k}\right) .
\end{aligned}
$$

The covariance tensor can alternatively be expressed in a matrix form as

$$
\operatorname{cov}(\operatorname{vec}(\mathbf{G}))=p \boldsymbol{\Sigma} \otimes \boldsymbol{\Sigma}+[p \boldsymbol{\Sigma} \otimes \boldsymbol{\Sigma}] \mathbf{K}_{n n}=\left(\mathbf{I}_{n^{2}}+\mathbf{K}_{n n}\right)(p \boldsymbol{\Sigma} \otimes \boldsymbol{\Sigma}) .
$$

The matrix $\mathbf{K}_{n n}$ is known as the commutation matrix ${ }^{1}$ or vec-permutation matrix ${ }^{2}$ of order $n^{2} \times n^{2}$. In Eq. (18), the values of $\bar{G}_{i k}$ etc. are fixed by the mean matrix. Therefore, the only parameter which controls the uncertainty in the distribution is $\theta$. This is a gross 'oversimplification' of the naturae of the uncertainty in some sense. Any $n \times n$ symmetric matrix $\mathbf{G}$, supposing fully populated, can have $N=n(n+1) / 2$ number of independent elements. Therefore, its covariance matrix is $N^{2}=n^{2}(n+1)^{2} / 4$ dimensional and symmetric. Which implies that it can have $N(N+1) / 2=n(n+1)(n(n+1)+2) / 8$ number of independent elements. Nonparametric approach, therefore, only offers a single parameter to quantify uncertainty which can potentially be expressed by $n(n+1)(n(n+1)+2) / 8$ number of independent parameters. However, when very little information regarding the covariance tensor of $\mathbf{G}$ is available, then a central Wishart distribution is the best one can use to quantify uncertainty. If more reliable information regarding the covariance tensor of $\mathbf{G}$ is available, then we need a matrix variate distribution which not only satisfy the constrains mentioned before, but also must offer more parameters to fit the 'known' covariance tensor of G. This is the central motivation of the work taken up in this paper. 


\section{Derivation of the Unified Distribution}

\section{A. Functional Form of the Distribution}

Because $\mathbf{G}$ is a symmetric and positive-definite random matrix, it can be always be factorized as

$$
\mathbf{G}=\mathbf{X} \mathbf{X}^{T}
$$

where $\mathbf{X} \in \mathbb{R}^{n \times p}, p \geq n$ is in general a rectangular matrix. Since the factorization in Eq. (20) will always (for $p \geq n$ ) guarantee the satisfaction of the the symmetry and the positive-definiteness condition, we assume that this is the form of the random system matrices arising in structural dynamics. Now we need to study the probabilistic nature of the random matrix $\mathbf{X}$. Once the pdf of $\mathbf{X}$ is known, the pdf $\mathbf{G}$ will be derived using the non-linear matrix transformation in Eq. (20).

Suppose the mean of $\mathbf{X}$ is $\mathcal{M} \in \mathbb{R}^{n \times p}, p \geq n$ and the covariance tensor of $\mathbf{X}$ is given by $\boldsymbol{\Sigma} \otimes \mathbf{I}_{p} \in \mathbb{R}^{n m \times n m}$ where $\boldsymbol{\Sigma} \in \mathbb{R}_{n}^{+}$. How to obtain $p, \mathcal{M}$ and $\boldsymbol{\Sigma}$ will be discussed later. At this point it is suffice to assume that the mean and covariance of the random matrix $\mathbf{X}$ exist. Suppose the matrix variate probability density function of $\mathbf{X} \in \mathbb{R}^{n \times p}$ is given by $p_{\mathbf{X}}(\mathbf{X}): \mathbb{R}^{n \times p} \rightarrow \mathbb{R}$. We have the following information and constrains to obtain $p_{\mathbf{X}}(\mathbf{X})$ :

$$
\begin{aligned}
& \int_{\mathbf{X} \in \mathbb{R}^{n \times p}} p_{\mathbf{X}}(\mathbf{X}) d \mathbf{X}=1 \quad \text { (normalization) } \\
& \int_{\mathbf{X} \in \mathbb{R}^{n \times p}} \mathbf{X} p_{\mathbf{X}}(\mathbf{X}) d \mathbf{X}=\mathcal{M} \quad \text { (the mean matrix) } \\
& \text { and } \quad \int_{\mathbf{X} \in \mathbb{R}^{n \times p}}[\mathbf{X}-\mathcal{M}] \otimes[\mathbf{X}-\mathcal{M}]^{T} p_{\mathbf{X}}(\mathbf{X}) d \mathbf{X}=\mathbf{\Sigma} \otimes \mathbf{I}_{p} \quad \text { (the covariance matrix). }
\end{aligned}
$$

The integrals appearing in the above three equations are $n \times p$ dimensional. Extending the standard maximum entropy argument to the matrix case we can say that the pdf of $\mathbf{X}$ is given by the matrix variate Gaussian distribution, that is, $\mathbf{X} \sim N_{n, p}\left(\mathcal{M}, \boldsymbol{\Sigma} \otimes \mathbf{I}_{p}\right)$.

We first derive the characteristic function of $\mathbf{G}=\mathbf{X X}^{T}$. Since $\mathbf{X} \sim N_{n, p}\left(\mathcal{M}, \boldsymbol{\Sigma} \otimes \mathbf{I}_{p}\right)$, a rectangular Gaussian random matrix with mean $\mathcal{M}$ and covariance matrix $\left[\boldsymbol{\Sigma} \otimes \mathbf{I}_{p}\right] \in \mathbb{R}^{n p \times n p}$, the probability density function of $\mathbf{X}$ can be obtained from Eq. (2) as

$$
p_{\mathbf{X}}(\mathbf{X})=(2 \pi)^{-n p / 2}|\boldsymbol{\Sigma}|^{-p / 2} \operatorname{etr}\left\{-\frac{1}{2} \boldsymbol{\Sigma}^{-1}(\mathbf{X}-\mathcal{M})(\mathbf{X}-\mathcal{M})^{T}\right\} .
$$

The matrix variate characteristic function of $\mathbf{G}$ can be defined as

$$
\phi_{\mathbf{G}}(\mathbf{Z})=\mathrm{E}[\operatorname{etr}\{-\mathbf{Z G}\}]=\int_{\mathbf{G}>0} \operatorname{etr}\{-\mathbf{Z G}\} p_{\mathbf{G}}(\mathbf{G}) d \mathbf{G}
$$

where $\mathbf{Z}$ is a symmetric matrix. Using the definition in (25) we have

$$
\phi_{\mathbf{G}}(\mathbf{Z})=\mathrm{E}[\operatorname{etr}\{-\mathbf{Z G}\}]=\mathrm{E}\left[\operatorname{etr}\left\{-\mathbf{Z X X} \mathbf{X}^{T}\right\}\right]
$$

Using the probability density function of $\mathbf{X}$ in Eq. (24) we have

$$
\phi_{\mathbf{G}}(\mathbf{Z})=(2 \pi)^{-n p / 2}|\boldsymbol{\Sigma}|^{-p / 2} \int_{\mathbb{R}^{n \times p}} \operatorname{etr}\left\{-\mathbf{Z X X} \mathbf{X}^{T}-\frac{1}{2} \boldsymbol{\Sigma}^{-1}(\mathbf{X}-\mathcal{M})(\mathbf{X}-\mathcal{M})^{T}\right\} d \mathbf{X} .
$$

The domain of the above integral is the space of $n \times p$ real matrices. This integral can be evaluated exactly to obtain

$$
\phi_{\mathbf{G}}(\mathbf{Z})=|\boldsymbol{\Sigma}|^{-p / 2}\left|2 \mathbf{Z}+\boldsymbol{\Sigma}^{-1}\right|^{-p / 2} \operatorname{etr}\left\{\frac{1}{2} \boldsymbol{\Theta}+\frac{1}{2} \boldsymbol{\Theta} \boldsymbol{\Sigma}^{-1}\left(2 \mathbf{Z}+\boldsymbol{\Sigma}^{-1}\right)^{-1}\right\}
$$

where $\Theta=\boldsymbol{\Sigma}^{-1} \mathcal{M} \mathcal{M}^{T}$. Taking the matrix variate inverse matrix Laplace transform of (28), it can be shown (see the books by Gupta and Nagar $^{29}$ and Muirhead ${ }^{27}$ for relevant mathematical details on matrix variate Laplace transforms) that the pdf of $\mathbf{G}$ follows the noncentral Wishart distribution given in Eq. (5), that is

$$
\begin{aligned}
p_{\mathbf{G}}(\mathbf{G})=\left\{2^{\frac{1}{2} n p} \Gamma_{n}\left(\frac{1}{2} p\right)|\boldsymbol{\Sigma}|^{\frac{1}{2} p}\right\}^{-1} \operatorname{etr}\left\{-\frac{1}{2} \boldsymbol{\Theta}\right\} \operatorname{etr}\left\{-\frac{1}{2} \boldsymbol{\Sigma}^{-1} \mathbf{G}\right\} & |\mathbf{S}|^{\frac{1}{2}(p-n-1)}{ }_{0} F_{1}\left(\frac{1}{2} p, \frac{1}{4} \boldsymbol{\Theta} \boldsymbol{\Sigma}^{-1} \mathbf{G}\right) .
\end{aligned}
$$


From the discussions so far, we have the following fundamental result regarding the unified uncertainty modelling of structural dynamic systems:

Theorem 1. The unified parametric-nonparametric probability density function a random system matrix $\mathbf{G} \equiv\{\mathbf{M}, \mathbf{C}, \mathbf{K}\}$ follows the noncentral Wishart distribution, that is $\mathbf{G} \sim W_{n}(p, \boldsymbol{\Sigma}, \boldsymbol{\Theta})$ where $p>n$ is a real scalar, $\boldsymbol{\Sigma}$ and $\boldsymbol{\Theta}$ are symmetric positive-definite $n \times n$ real matrices.

If the noncentrality parameter $\Theta$ is a null matrix, the unified distribution reduces to the nonparametric distribution (central Wishart distribution). The unified distribution derived here is therefore further generalization of the nonparametric distribution. The additional parameters provided by the matrix $\Theta \in \mathbb{R}_{n}^{+}$allow to model parametric uncertainty which is not available within the scope of the nonparametric distribution.

\section{B. Properties of the Distribution}

For notational convenience, suppose

$$
\boldsymbol{\Omega}=\mathcal{M} \mathcal{M}^{T}
$$

Therefore,

$$
\Theta=\boldsymbol{\Sigma}^{-1} \mathcal{M} \mathcal{M}^{T}=\boldsymbol{\Sigma}^{-1} \boldsymbol{\Omega}
$$

that is

$$
\Omega=\Sigma \Theta .
$$

The first moment (mean), the second-moment and the elements of the covariance tensor can be obtained ${ }^{29}$ as

$$
\begin{aligned}
& \mathrm{E}[\mathbf{G}]=p \boldsymbol{\Sigma}+\boldsymbol{\Omega}, \\
& \mathrm{E}\left[\mathbf{G}^{2}\right]=p \operatorname{Trace}(\boldsymbol{\Sigma}) \boldsymbol{\Sigma}+p \boldsymbol{\Sigma}^{2}+p^{2} \boldsymbol{\Sigma}^{2}+p \boldsymbol{\Omega} \boldsymbol{\Sigma}+\boldsymbol{\Omega} \boldsymbol{\Sigma} \\
& \\
& \quad+\operatorname{Trace}(\boldsymbol{\Omega}) \boldsymbol{\Sigma}+\operatorname{Trace}(\boldsymbol{\Sigma}) \boldsymbol{\Omega}+\boldsymbol{\Sigma} \boldsymbol{\Omega}+p \boldsymbol{\Sigma} \boldsymbol{\Omega}+\boldsymbol{\Omega}^{2}, \\
& \text { and } \quad \operatorname{cov}\left(G_{i j}, G_{k l}\right)=\left(p \Sigma_{i j}+\Omega_{i j}\right)\left(p \Sigma_{k l}+\Omega_{k l}\right) \\
& \quad+p\left(\Sigma_{i k} \Sigma_{j l}+\Sigma_{i l} \Sigma_{j k}\right)+\left(\Sigma_{j l} \Omega_{i k}+\Sigma_{i l} \Omega_{j k}+\Sigma_{j k} \Omega_{i l}+\Sigma_{i k} \Omega_{j l}\right) .
\end{aligned}
$$

The covariance tensor can alternatively be expressed in a matrix form as

$$
\begin{aligned}
\operatorname{cov}(\operatorname{vec}(\mathbf{G})) & =p \boldsymbol{\Sigma} \otimes \boldsymbol{\Sigma}+\boldsymbol{\Omega} \otimes \boldsymbol{\Sigma}+\boldsymbol{\Sigma} \otimes \boldsymbol{\Omega}+[p \boldsymbol{\Sigma} \otimes \boldsymbol{\Sigma}+\boldsymbol{\Omega} \otimes \boldsymbol{\Sigma}+\boldsymbol{\Sigma} \otimes \boldsymbol{\Omega}] \mathbf{K}_{n n} \\
& =\left(\mathbf{I}_{n^{2}}+\mathbf{K}_{n n}\right)(p \boldsymbol{\Sigma} \otimes \boldsymbol{\Sigma}+\boldsymbol{\Omega} \otimes \boldsymbol{\Sigma}+\boldsymbol{\Sigma} \otimes \boldsymbol{\Omega}) .
\end{aligned}
$$

Comparing these expressions with the equivalent expressions (17)-(19) corresponding to the nonparametric distribution in section III, observe that the unified distribution offers more parameters to 'capture' the parametric nature of uncertainty.

\section{Parameter Identification of the Distribution}

The unknown parameters of the unified distribution are $p \in \mathbb{R}, \boldsymbol{\Sigma} \in \mathbb{R}_{n}^{+}$and $\boldsymbol{\Omega} \in \mathbb{R}_{n}^{+}$. There are in total $n+n(n+1)$ number of unknowns in this distribution. A least-square error minimization approach based on the expressions of the mean and covariance matrix in Eqs. (33) and (36) is developed in this part.

Before proceeding to the parameter identification of the matrix variate distribution, let us look at the information an analyst is likely to have. The mean of system matrices can be considered as the baseline finite element model so that we have $\overline{\mathbf{G}} \in \mathbb{R}_{n}^{+}$. For systems with uncertain parameters we can also obtain the covariance matrix of $\mathbf{G}$, denoted by

$$
\mathbf{C}_{G}=\operatorname{cov}(\operatorname{vec}(\mathbf{G})) \in \mathbb{R}_{n^{2}}^{+} .
$$

If a random field model is assumed for the system parameters, then the covariance matrix can be obtained by discretising the random fields using the well established stochastic finite element approach ${ }^{6-15}$ (e.g., using the Karhunen-Loéve expansion). However, in general it is difficult to obtain the correlation function and other information regarding the random fields describing the system parameters accurately and reliably. Therefore, the elements of the covariance matrix $\mathbf{C}_{G}$ of the system matrix $\mathbf{G}$ are, at best, good approximations. Error in the elements of the covariance matrix arises not because of the numerical methods to obtain them, but due to the lack of knowledge regarding uncertainty in the system. As a result, every element of the covariance matrix $\mathbf{C}_{G}$ may not be very reliable for practical problems. Consequently here we satisfy them in a least-square sense and not in an'element-by-element' basis. 
Nonparametric analysis only considers the trace of $\mathbf{C}_{G}$ and therefore neglects all the non-diagonal elements. Here we aim to consider the complete covariance matrix in a least-square sense. The idea behind the lest-square satisfaction is steaming from the fact that the elements of $\mathbf{C}_{G}$ are only accurate to the extent of underlying information available regarding the detailed nature of uncertainty (e.g, correlation functions, correlation lengths, standard deviations of the uncertain parameters). With the least-square satisfaction of the covariance matrix, combined with the random matrix model, we hope to take both parametric and non-parametric uncertainty into account simultaneously.

The mean of a systems matrix, $\overline{\mathbf{G}}$, is symmetric and positive definite. From Eq. (33) we have

$$
\begin{array}{r}
p \boldsymbol{\Sigma}+\boldsymbol{\Omega}=\overline{\mathbf{G}} \\
\text { or } \quad \boldsymbol{\Sigma}=(\overline{\mathbf{G}}-\boldsymbol{\Omega}) / p
\end{array}
$$

Substituting $\Sigma$ into the expression of the covariance matrix in Eq. (36) and simplifying one obtains

$$
\operatorname{cov}(\operatorname{vec}(\mathbf{G}))=\left(\mathbf{I}_{n^{2}}+\mathbf{K}_{n n}\right) \frac{1}{p}(\overline{\mathbf{G}} \otimes \overline{\mathbf{G}}-\mathbf{\Omega} \otimes \mathbf{\Omega}) .
$$

We aim to obtain the normalized standard deviation defined in Eq. (13). First note that

$$
\begin{aligned}
\mathrm{E}\left[\|\mathbf{G}-\mathrm{E}[\mathbf{G}]\|_{\mathrm{F}}^{2}\right] & =\mathrm{E}\left[\operatorname{Trace}\left([\mathbf{G}-\mathrm{E}[\mathbf{G}]][\mathbf{G}-\mathrm{E}[\mathbf{G}]]^{T}\right)\right] \\
& =\operatorname{Trace}\left(\mathrm{E}\left[[\mathbf{G}-\mathrm{E}[\mathbf{G}]][\mathbf{G}-\mathrm{E}[\mathbf{G}]]^{T}\right]\right) \\
& =\operatorname{Trace}(\operatorname{cov}(\operatorname{vec}(\mathbf{G}))) \\
& =\operatorname{Trace}\left(\left(\mathbf{I}_{n^{2}}+\mathbf{K}_{n n}\right) \frac{1}{p}(\overline{\mathbf{G}} \otimes \overline{\mathbf{G}}-\boldsymbol{\Omega} \otimes \boldsymbol{\Omega})\right) \\
& =\frac{1}{p}\left(\operatorname{Trace}\left(\left(\mathbf{I}_{n^{2}}+\mathbf{K}_{n n}\right)(\overline{\mathbf{G}} \otimes \overline{\mathbf{G}})\right)-\operatorname{Trace}\left(\left(\mathbf{I}_{n^{2}}+\mathbf{K}_{n n}\right)(\boldsymbol{\Omega} \otimes \boldsymbol{\Omega})\right)\right) \\
& =\frac{1}{p}\left(\operatorname{Trace}\left(\overline{\mathbf{G}}^{2}\right)+\{\operatorname{Trace}(\overline{\mathbf{G}})\}^{2}-\left(\operatorname{Trace}\left(\boldsymbol{\Omega}^{2}\right)+\{\operatorname{Trace}(\boldsymbol{\Omega})\}^{2}\right)\right) .
\end{aligned}
$$

In calculating the preceding expression we have interchanged the $\mathrm{E}[\bullet]$ and Trace $(\bullet)$ as they linear operators and used Theorem 16.4.1 in Harville. ${ }^{2}$ Therefore

$$
\delta_{G}^{2}=\frac{\mathrm{E}\left[\|\mathbf{G}-\mathrm{E}[\mathbf{G}]\|_{\mathrm{F}}^{2}\right]}{\|\mathrm{E}[\mathbf{G}]\|_{\mathrm{F}}^{2}}=\frac{\operatorname{Trace}\left(\overline{\mathbf{G}}^{2}\right)+\{\operatorname{Trace}(\overline{\mathbf{G}})\}^{2}-\left(\operatorname{Trace}\left(\boldsymbol{\Omega}^{2}\right)+\{\operatorname{Trace}(\boldsymbol{\Omega})\}^{2}\right)}{p \operatorname{Trace}\left(\overline{\mathbf{G}}^{2}\right)} .
$$

From this expressions we obtain

$$
p=\frac{1}{\delta_{G}^{2}} \frac{\operatorname{Trace}\left(\overline{\mathbf{G}}^{2}-\mathbf{\Omega}^{2}\right)+\{\operatorname{Trace}(\overline{\mathbf{G}})\}^{2}-\{\operatorname{Trace}(\boldsymbol{\Omega})\}^{2}}{\operatorname{Trace}\left(\overline{\mathbf{G}}^{2}\right)} .
$$

In the above expression $\Omega \in \mathbb{R}^{n}$ is unknown. For the central Wishart distribution, $\Omega$ is a null matrix. We are trying to identify a non-null $\Omega$ matrix for the noncentral distribution.

For any symmetric matrix $\mathbf{G}$, one has

$$
\operatorname{vech}(\mathbf{G})=\mathbf{H}_{n} \operatorname{vec}(\mathbf{G})
$$

where $\mathbf{H}_{n}$ is the left Moore-Penrose inverse of the duplication matrix $\mathbf{G}_{n}$ (see Chapter 16 of the book by Harville ${ }^{2}$ ). From this we have

$$
\operatorname{cov}(\operatorname{vech}(\mathbf{G}))=\operatorname{cov}\left(\mathbf{H}_{n} \operatorname{vec}(\mathbf{G})\right) \operatorname{E}\left[\mathbf{H}_{n} \operatorname{vec}(\mathbf{G}) \operatorname{vec}(\mathbf{G})^{T} \mathbf{H}_{n}^{T}\right]=\mathbf{H}_{n} \operatorname{cov}(\operatorname{vec}(\mathbf{G})) \mathbf{H}_{n}^{T} .
$$

Substituting the expression of the covariance matrix from Eq. (40) into the preceding expression and using the derivations in Section 16.4 in Harville ${ }^{2}$ we have

$$
\begin{aligned}
\operatorname{cov}(\operatorname{vech}(\mathbf{G})) & =\mathbf{H}_{n} \operatorname{cov}(\operatorname{vec}(\mathbf{G})) \mathbf{H}_{n}^{T}=\mathbf{H}_{n}\left(\mathbf{I}_{n^{2}}+\mathbf{K}_{n n}\right) \frac{1}{p}(\overline{\mathbf{G}} \otimes \overline{\mathbf{G}}-\boldsymbol{\Omega} \otimes \boldsymbol{\Omega}) \mathbf{H}_{n}^{T} \\
& =\frac{2}{p} \mathbf{H}_{n}(\overline{\mathbf{G}} \otimes \overline{\mathbf{G}}-\boldsymbol{\Omega} \otimes \boldsymbol{\Omega}) \mathbf{H}_{n}^{T} .
\end{aligned}
$$


We suppose that $\mathbf{C}_{G}=\operatorname{cov}(\operatorname{vec}(\mathbf{G})) \in \mathbb{R}_{n^{2}}^{+}$is known. Using the relationship in Eq. (45), and equating the known covariance matrix to (46) we have

$$
\begin{array}{cc} 
& \mathbf{H}_{n} \mathbf{C}_{G} \mathbf{H}_{n}^{T}=\frac{2}{p} \mathbf{H}_{n}(\overline{\mathbf{G}} \otimes \overline{\mathbf{G}}-\boldsymbol{\Omega} \otimes \boldsymbol{\Omega}) \mathbf{H}_{n}^{T} \\
\text { or } & \mathbf{H}_{n}\left[\mathbf{C}_{G}-\frac{2}{p}(\overline{\mathbf{G}} \otimes \overline{\mathbf{G}}-\boldsymbol{\Omega} \otimes \boldsymbol{\Omega})\right] \mathbf{H}_{n}^{T}=\mathbf{O}_{n(n+1) / 2, n(n+1) / 2}
\end{array}
$$

Since the rank of $\mathbf{H}_{n}$ is $n(n+1) / 2$, the above equation will be satisfied if

$$
\begin{array}{ll} 
& \mathbf{C}_{G}=\frac{2}{p}(\overline{\mathbf{G}} \otimes \overline{\mathbf{G}}-\boldsymbol{\Omega} \otimes \boldsymbol{\Omega}) \\
\text { or } & \boldsymbol{\Omega} \otimes \boldsymbol{\Omega}=\overline{\mathbf{G}} \otimes \overline{\mathbf{G}}-p \mathbf{C}_{G} / 2 .
\end{array}
$$

We need to solve this equation to obtain $\boldsymbol{\Omega}$. The solution of $\boldsymbol{\Omega}$ will contain $p$ and as noted in Eq. (43), $p$ in turn is expressed in terms of $\boldsymbol{\Omega}$. Therefore some iterative type solution method is required to obtain both the parameters.

Supposing $\mathbf{G}$ is fully populated $\mathbf{C}_{G}$ can have $n(n+1)(n(n+1)+2) / 8$ number of independent elements. Whereas $\boldsymbol{\Omega}$ has only $n(n+1) / 2$ number of independent elements. Clearly an unique solution of $\boldsymbol{\Omega}$ cannot be obtained. Here a least-square error minimization method is adopted. Specifically we are aiming to identify $\boldsymbol{\Omega}$ such that the following norm

$$
\|\mathcal{A}-\boldsymbol{\Omega} \otimes \boldsymbol{\Omega}\|_{\mathrm{F}}
$$

where

$$
\mathcal{A}=\overline{\mathbf{G}} \otimes \overline{\mathbf{G}}-p \mathbf{C}_{G} / 2 \in \mathbb{R}^{n^{2} \times n^{2}}
$$

is minimized. The problem of identifying general real matrices $\boldsymbol{\Omega}_{1}$ and $\boldsymbol{\Omega}_{2}$ which minimizes $\left\|\mathcal{A}-\boldsymbol{\Omega}_{1} \otimes \boldsymbol{\Omega}_{2}\right\|_{\mathrm{F}}$ was first discussed by Van Loan and Pitsianis. ${ }^{30}$ This problem is known as the 'nearest Kronecker product'. We refer to the papers by Langville and Stewart ${ }^{31-33}$ for further discussions. More recently Van Loan [personal communication] provided an elegant solution of problem (51) which is a constrained version of the general nearest Kronecker product problem discussed in the earlier paper. ${ }^{30}$

From the discussion so far we can say that the unified parametric-nonparametric distribution of a structural matrix can be expressed by a noncentral Wishart distribution so that $\mathbf{G} \sim W_{n}(p, \boldsymbol{\Sigma}, \boldsymbol{\Theta})$. The parameters of the distribution, namely $p \in \mathbb{R}, \boldsymbol{\Sigma} \in \mathbb{R}_{n}^{+}$and $\boldsymbol{\Omega} \in \mathbb{R}_{n}^{+}$can be calculated by following these steps:

1. Obtain the mean matrix (corresponding to the baseline system) $\overline{\mathbf{G}} \in \mathbb{R}_{n}^{+}$from the standard FE analysis. Obtain the covariance matrix $\mathbf{C}_{G}=\operatorname{cov}(\operatorname{vec}(\mathbf{G})) \in \mathbb{R}_{n^{2}}^{+}$corresponding to $\overline{\mathbf{G}}$ (e. g., using the Kerhunen Love expansion ${ }^{7}$ of the parametric random fields). The elements of $\mathbf{C}_{G}$ do not have to be very accurate (with the understanding that inaccuracies are arising due to the lack knowledge regarding the parametric stochastic fields).

2. Obtain the normalized standard deviation $\delta_{G}$ of $\mathbf{G}$ from

$$
\delta_{G}^{2}=\frac{\mathrm{E}\left[\|\mathbf{G}-\mathrm{E}[\mathbf{G}]\|_{\mathrm{F}}^{2}\right]}{\|\mathrm{E}[\mathbf{G}]\|_{\mathrm{F}}^{2}}=\frac{\operatorname{Trace}\left(\mathbf{C}_{G}\right)}{\operatorname{Trace}\left(\overline{\mathbf{G}}^{2}\right)} .
$$

3. Begin with $\boldsymbol{\Omega}=\mathbf{O}_{n, n}$ (as in the case of non-parametric distribution) and obtain the initial value of $p$ from Eq. (43) as

$$
p=\frac{1}{\delta_{G}^{2}} \frac{\operatorname{Trace}\left(\overline{\mathbf{G}}^{2}\right)+\{\operatorname{Trace}(\overline{\mathbf{G}})\}^{2}}{\text { Trace }\left(\overline{\mathbf{G}}^{2}\right)} .
$$

From the discussion in section III, note that this value of $p$ is used in the non-parametric approach. The steps below are aimed at incorporating parametric uncertainties.

4. Form the matrix $\mathcal{A}=\overline{\mathbf{G}} \otimes \overline{\mathbf{G}}-p \mathbf{C}_{G} / 2 \in \mathbb{R}^{n^{2} \times n^{2}}$ and obtain $\boldsymbol{\Omega} \in \mathbb{R}^{n \times n}$ by least-square minimization of the Frobenius norm

$$
\|\mathcal{A}-\boldsymbol{\Omega} \otimes \boldsymbol{\Omega}\|_{\mathrm{F}} .
$$


5. Using this value of $\Omega \in \mathbb{R}^{n \times n}$, calculate the new value of $p$ from Eq. (43) as

$$
p_{\text {new }}=\frac{1}{\delta_{G}^{2}} \frac{\operatorname{Trace}\left(\overline{\mathbf{G}}^{2}-\boldsymbol{\Omega}^{2}\right)+\{\operatorname{Trace}(\overline{\mathbf{G}})\}^{2}-\{\operatorname{Trace}(\boldsymbol{\Omega})\}^{2}}{\operatorname{Trace}\left(\overline{\mathbf{G}}^{2}\right)} .
$$

6. Calculate $\delta p=\left|p-p_{\text {new }}\right| / n$

(a) If $\delta p \leq \epsilon_{p}\left(\epsilon_{p}\right.$ is a small number, say $\left.\epsilon_{p}=0.01\right)$ then the iteration has converged. Select $p=p_{\text {new }}$ and exit.

(b) Else if $\delta p>\epsilon_{p}$ then the iteration steps. Select $p=p_{\text {new }}$ and go back to step 4.

7. Finally calculate

$$
\boldsymbol{\Sigma}=(\overline{\mathbf{G}}-\mathbf{\Omega}) / p
$$

and

$$
\Theta=\Sigma^{-1} \Omega
$$

This iterative procedure completely defines all the unknown parameters in the noncentral Wishart distribution.

\section{Numerical Implementation of the Unified Model}

The parameters of the distribution $\mathbf{G} \sim W_{n}(p, \boldsymbol{\Sigma}, \boldsymbol{\Theta})$, namely $p \in \mathbb{R}, \boldsymbol{\Sigma} \in \mathbb{R}_{n}^{+}$and $\boldsymbol{\Omega} \in \mathbb{R}_{n}^{+}$can be calculated by following the method outlined in the previous section. In this section we outline a numerical procedure to generate the samples of the system matrix satisfying the unified distribution. The proposed method is based on the derivation itself, as described in subsection A

The simulation procedure is surprisingly simple and hinges on the factorization in Eq. (20). Basically one only needs to simulate a Gaussian random matrix $\mathbf{X} \sim N_{n, p}\left(\mathcal{M}, \boldsymbol{\Sigma} \otimes \mathbf{I}_{p}\right)$ and use this equation to generate the samples of the system matrix. This can be achieved by following these simple steps

1. Obtain the distribution parameters $p \in \mathbb{R}, \boldsymbol{\Sigma} \in \mathbb{R}_{n}^{+}$and $\boldsymbol{\Omega} \in \mathbb{R}_{n}^{+}$from $\overline{\mathbf{G}}$ and $\mathbf{C}_{G}$ using the iterative procedure described in the previous section. Approximate $p$ to its nearest integer (because $p$ is in the order of several thousands, this introduces very little error).

2. Perform the Cholesky factorizations of the positive definite matrices $\boldsymbol{\Sigma} \in \mathbb{R}_{n}^{+}$and $\Omega \in \mathbb{R}_{n}^{+}$as

$$
\begin{aligned}
\boldsymbol{\Sigma} & =\mathbf{D D}^{T}, \quad \mathbf{D} \in \mathbb{R}^{n \times n} \\
\text { and } \quad \boldsymbol{\Omega} & =\widehat{\mathcal{M}} \widehat{\mathcal{M}}^{T}, \quad \widehat{\mathcal{M}} \in \mathbb{R}^{n \times n} .
\end{aligned}
$$

3. Calculate the $n \times n$ square matrix

$$
\widetilde{\mathcal{M}}=\mathbf{D}^{-1} \widehat{\mathcal{M}}
$$

4. Construct the $n \times p$ rectangular mean matrix

$$
\mathcal{M}=\left[\widetilde{\mathcal{M}}, \mathbf{O}_{n, n-p}\right] \in \mathbb{R}^{n \times p}
$$

5. Obtain the matrix $\mathbf{Y} \in \mathbb{R}^{n \times p}$ containing uncorrelated Gaussian random numbers with mean $\mathcal{M}$ and unit standard deviation.

6. Generate the samples of a system matrix as

$$
\mathbf{G}=\mathbf{D Y} \mathbf{Y}^{T} \mathbf{D}^{T} \in \mathbb{R}_{n}^{+} .
$$

One needs to repeat the above procedure for the mass, stiffness and damping matrices. The above procedure can be implemented very easily. For example, in MATLAB ${ }^{\circledR}$, the following four lines of code will generate the samples of the system matrices: 


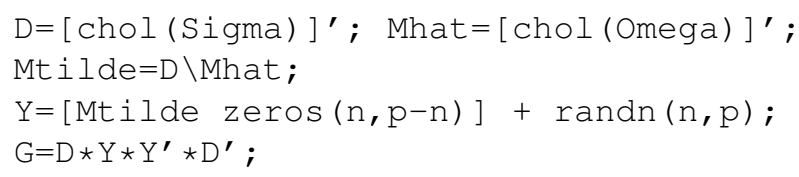

Once the samples of the system matrices are generated, the rest of the analysis is identical to any Monte Carlo simulation based approach. In the next section the proposed approach is illustrated thorough an example.

\section{Numerical Example: Dynamic Response of a Clamped Plate With Randomly Inhomogeneous Properties}

A cantilever steel plate with uncertain properties is considered to illustrate the application of the derived unified distribution in probabilistic structural dynamics. The diagram of the plate together with the deterministic numerical values assumed for the system parameters are shown in Fig. 2. The plate is excited by an unit harmonic force and the response is calculated at the point shown in the diagram. The standard four-noded thin plate bending element

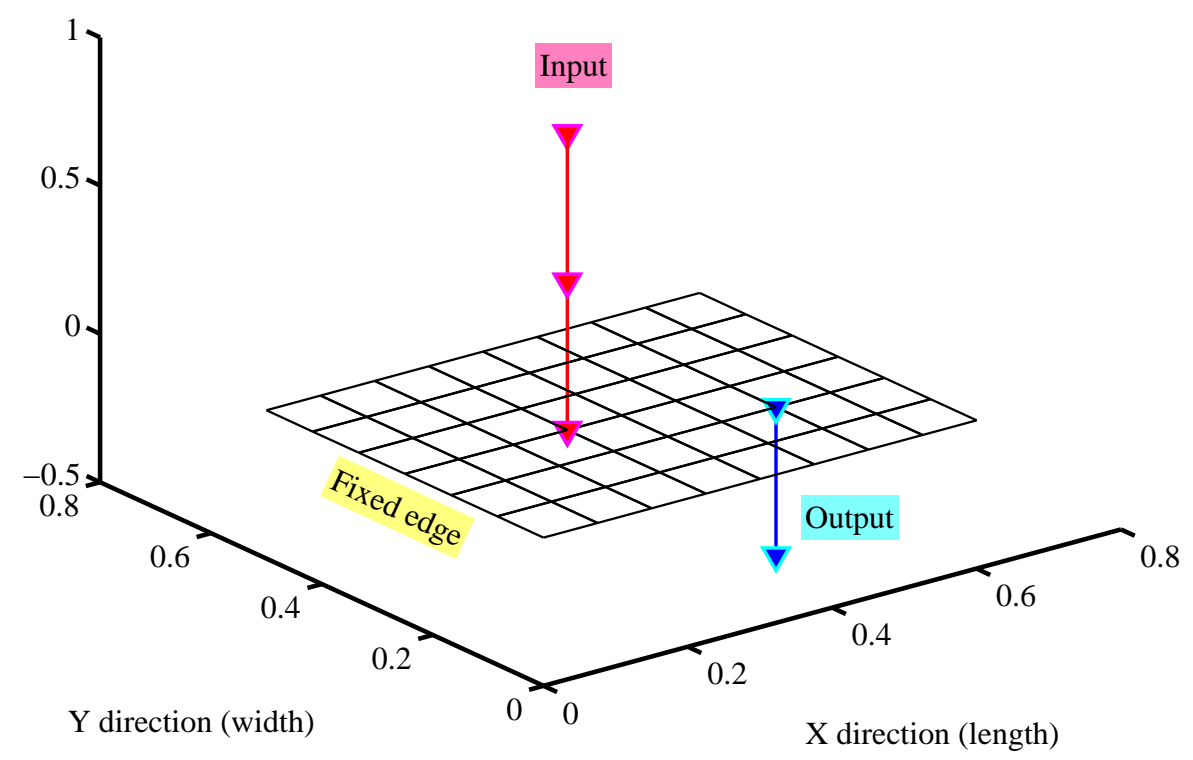

Figure 2. The Finite Element (FE) model of a steel cantilever plate: $25 \times 15$ elements, 416 nodes, 1200 degrees-of-freedom. The deterministic properties are: $\bar{E}=200 \times 10^{9} \mathbf{N} / \mathbf{m}^{2}, \bar{\mu}=0.3, \bar{\rho}=7860 \mathbf{k g} / \mathbf{m}^{3}, \bar{t}=3.0 \mathbf{m m}, L_{x}=0.6 \mathbf{m}, L_{y}=0.5 \mathrm{~m}$. Input node number: 79, output node number: 112 , modal damping factor: $2 \%$ for all modes.

(resulting 12 degrees of freedom per element) is used. The plate is divided into 8 elements along the $\mathrm{x}$-axis and 6 elements along the y-axis for the numerical calculations. The resulting system has 168 degrees of freedom so that $n=168$. It is assumed that the Young's modulus, Poissons ratio, mass density and thickness are random fields of the form

$$
\begin{aligned}
E(\mathbf{x}) & =\bar{E}\left(1+\epsilon_{E} f_{1}(\mathbf{x})\right) \\
\mu(\mathbf{x}) & =\bar{\mu}\left(1+\epsilon_{\mu} f_{2}(\mathbf{x})\right) \\
\rho(\mathbf{x}) & =\bar{\rho}\left(1+\epsilon_{\rho} f_{3}(\mathbf{x})\right) \\
\text { and } \quad t(\mathbf{x}) & =\bar{t}\left(1+\epsilon_{t} f_{4}(\mathbf{x})\right) .
\end{aligned}
$$

The two dimensional vector $\mathbf{x}$ denotes the spatial coordinates. The strength parameters are assumed to be $\epsilon_{E}=0.15$, $\epsilon_{\mu}=0.10, \epsilon_{\rho}=0.15$ and $\epsilon_{t}=0.15$. The random fields $f_{i}(\mathbf{x}), i=1, \cdots, 4$ are assumed to be delta-correlated homogenous Gaussian random fields. A 600-sample Monte Carlo simulation is performed to obtain the FRFs.

Direct stochastic finite-element Monte Carlo Simulation of the amplitude and phase of the cross-FRF are shown in Figs. 3 and 4 respectively. The realizations of the amplitude and phase of the FRF for each sample are shown

$$
11 \text { of } 22
$$




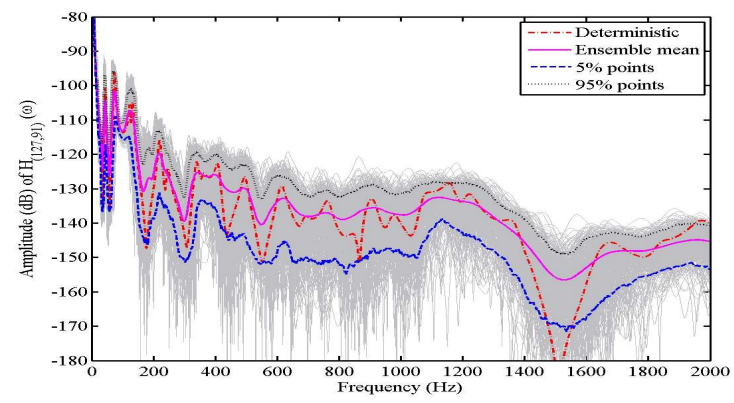

(a) Amplitude across the frequency range

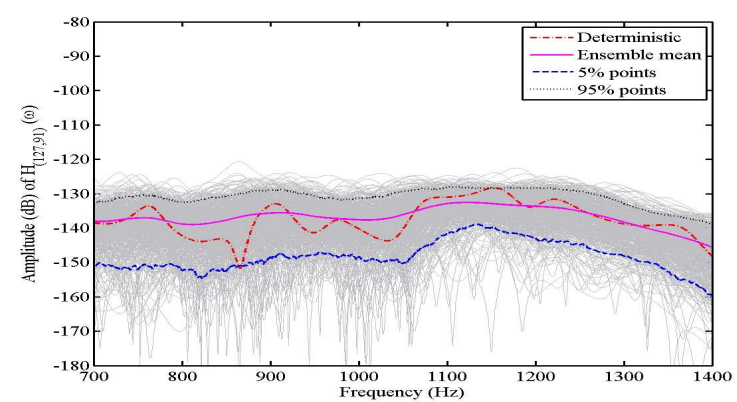

(c) Amplitude in the Medium-frequency range

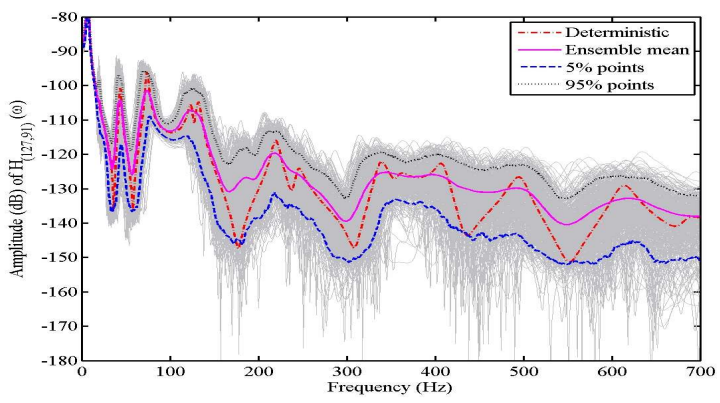

(b) Amplitude in the Low-frequency range

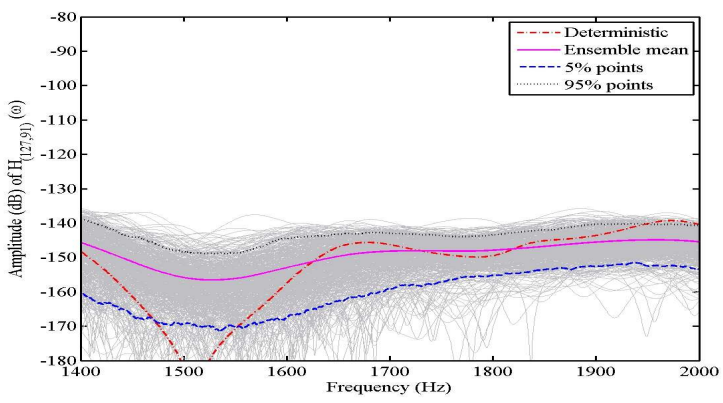

(d) Amplitude in the High-frequency range

Figure 3. Direct stochastic finite-element Monte Carlo Simulation of the amplitude of the cross-FRF of the plate with randomly distributed material properties.

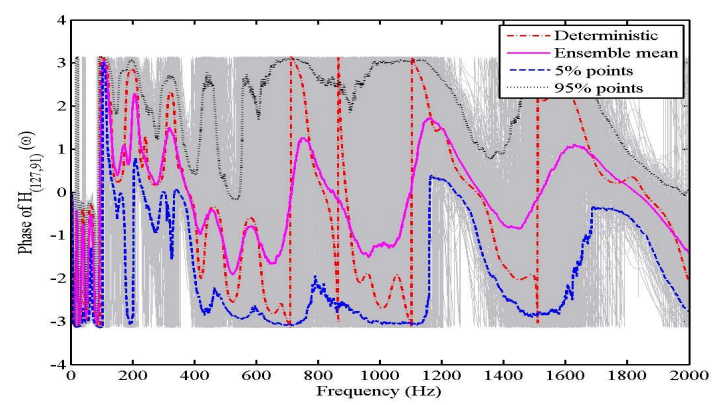

(a) Phase across the frequency range

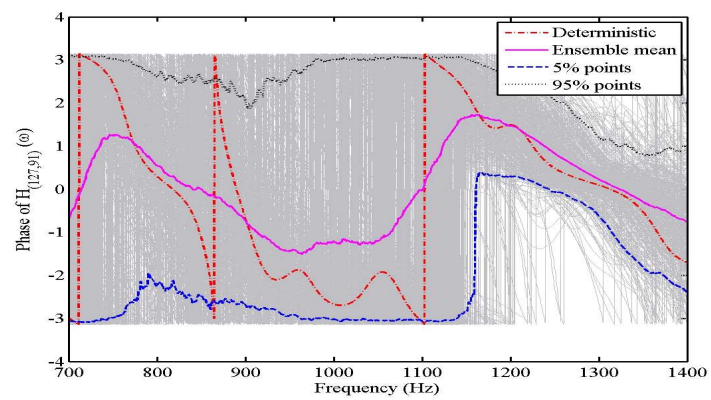

(c) Phase in the Medium-frequency range

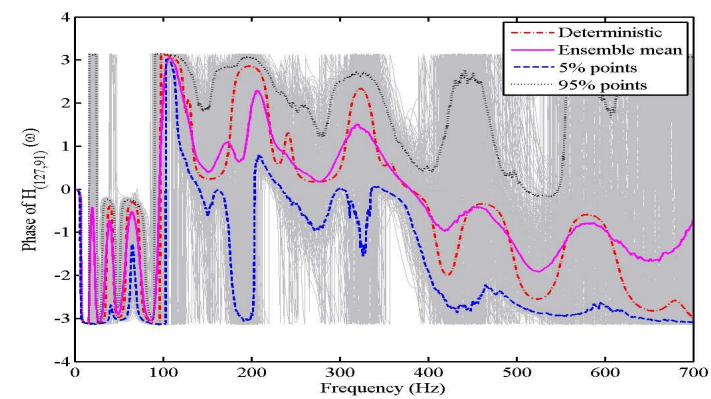

(b) Phase in the Low-frequency range

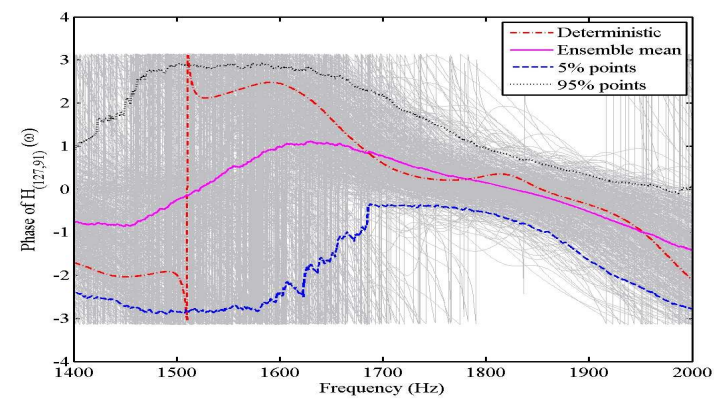

(d) Phase in the High-frequency range

Figure 4. Direct stochastic finite-element Monte Carlo Simulation of the phase of the cross-FRF of the plate with randomly distributed material properties. 
together with the ensemble mean, $5 \%$ and $95 \%$ probability points and the results for the baseline system. In Figs. 3 b)-(d) and 4(b)-(d) we have separately shown the low, medium and high-frequency response, obtained by zooming in the appropriate frequency ranges in Figs. 3 a) and 4(a) respectively. There are of course no fixed and definite boundaries between the low, medium and high-frequency ranges. We have selected $0-0.7 \mathrm{KHz}$ as the low-frequency range, $0.7-1.4 \mathrm{KHz}$ as the medium-frequency range and $1.4-2.0 \mathrm{KHz}$ as the high-frequency range. These frequency boundaries are selected on the basis of the qualitative nature of the response and devised purely for the clarity in presenting the results. The proposed noncentral Wishart matrix approach is independent of these selections. The ensemble mean of the response amplitude follows the deterministic result closely in the low and medium frequency ranges. However, the ensemble mean of the phase of the response is quite different from the deterministic result in the medium and high frequency ranges. Overall the spread in the amplitude and phase of the response increases with increasing frequency. The direct stochastic finite-element Monte Carlo Simulation of the amplitude and phase of the driving-point-FRF are shown in Figs. 5$]$ and 6 respectively. The realizations of the amplitude and phase of the

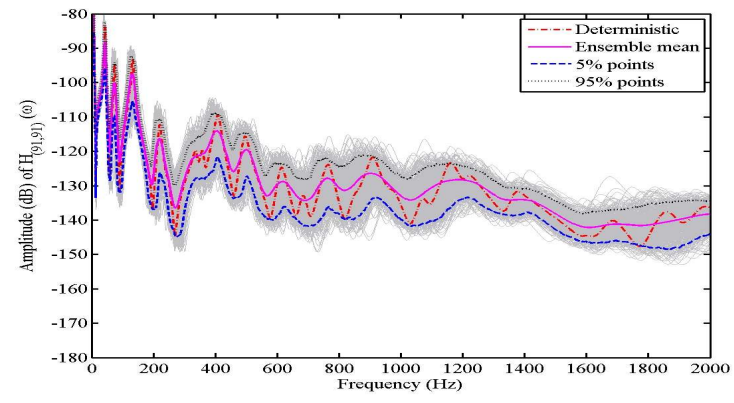

(a) Amplitude across the frequency range

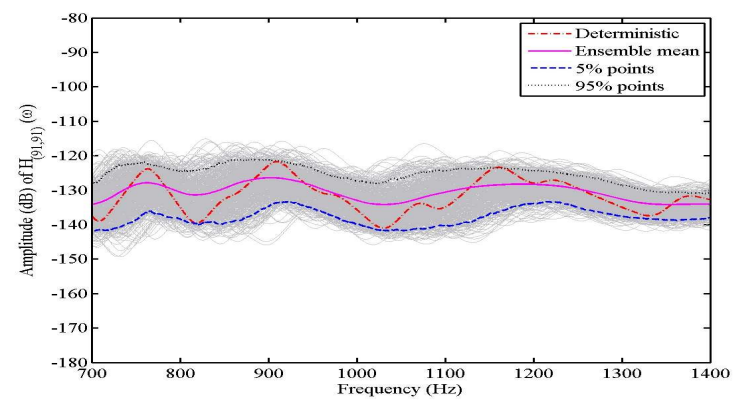

(c) Amplitude in the Medium-frequency range

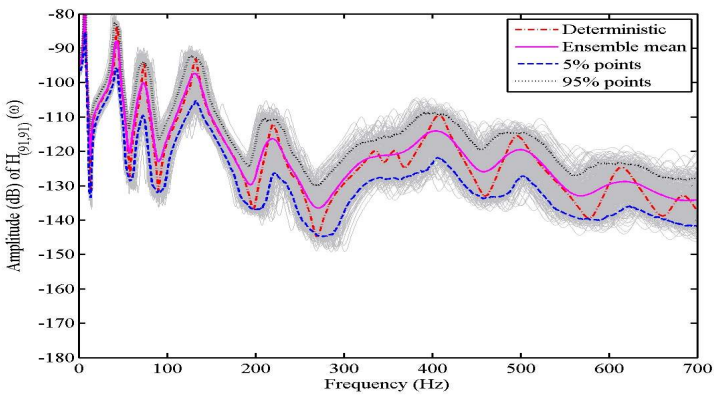

(b) Amplitude in the Low-frequency range

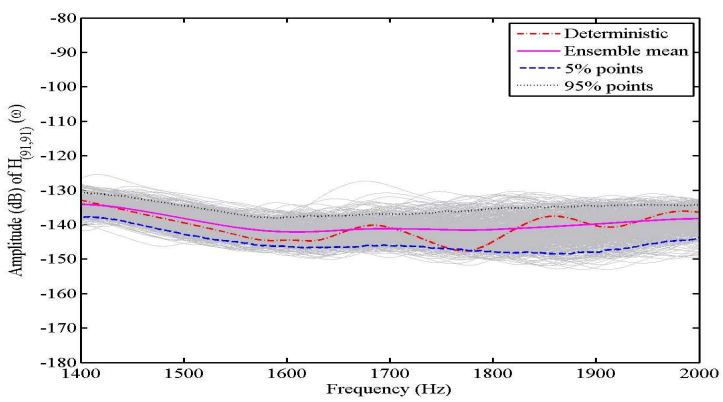

(d) Amplitude in the High-frequency range

Figure 5. Direct stochastic finite-element Monte Carlo Simulation of the amplitude of the driving-point-FRF of the plate with randomly distributed material properties.

FRF for each sample are shown together with the ensemble mean, 5\% and 95\% probability points and the results for the baseline system. In Figs. 5(b)-(d) and 6(b)-(d) we have separately shown the low, medium and high-frequency response, obtained by zooming in the appropriate frequency ranges in Figs. 5(a) and 6/a) respectively. The ensemble mean follows the deterministic result closely across the frequency range. The amount of spread in the amplitude and phase of the response in this case is smaller compared to the cross-FRF. This is due to the fact that the affect of distributed random material properties is more when we measure the response at a point further to the source.

Now we want to see if the results obtained from the direct stochastic finite element Monte Carlo Simulation can be reproduced using noncentral Wishart random matrices whose parameters are identified according to the method outlined before. We want to see whether the proposed method can predict the known variability in the response in the case when very accurate information regarding parametric uncertainty is available. The purpose of this is simply to validate the proposed formulation. The intended use of the proposed approach is however for the case when the elements of the covariance matrix of the system matrices are not very accurate. The details of the parametric variations of the random fields will not be used in the noncentral Wishart matrix approach. From the simulated random mass and stiffness matrices we obtain we obtain $\mathbf{C}_{M}, \mathbf{C}_{K}, n=168, \delta_{M}=0.1194$ and $\delta_{K}=0.2711$. Since $2 \%$ constant modal damping factor is assumed for all the modes, $\delta_{C}=0$ and $\mathbf{C}_{C}=\mathbf{O}_{n^{2}, n^{2}}$. The only uncertainty related information used in the random matrix approach are the values of $\mathbf{C}_{M}, \mathbf{C}_{K}, \delta_{M}$ and $\delta_{K}$. The detailed information regarding which 


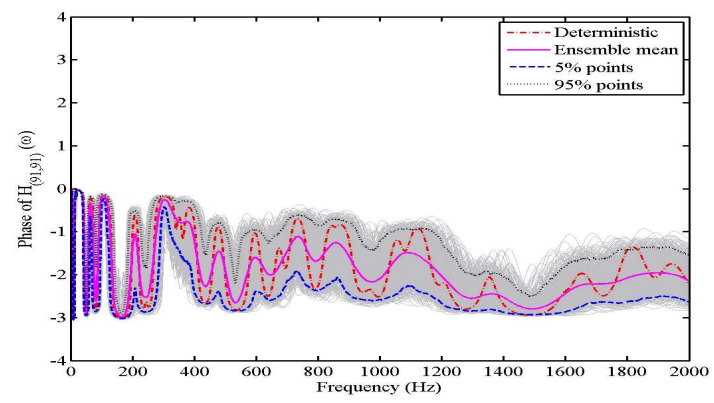

(a) Phase across the frequency range

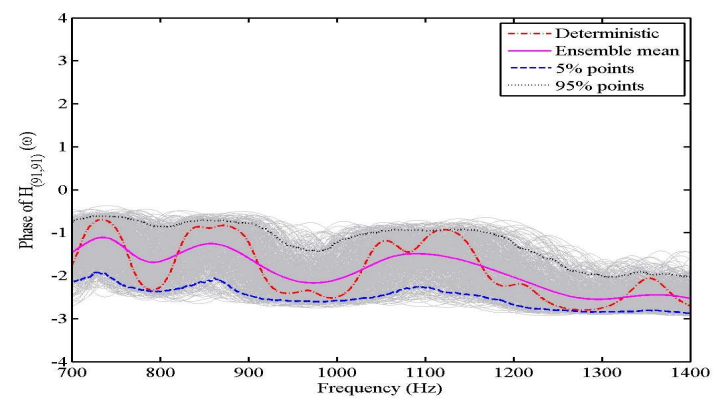

(c) Phase in the Medium-frequency range

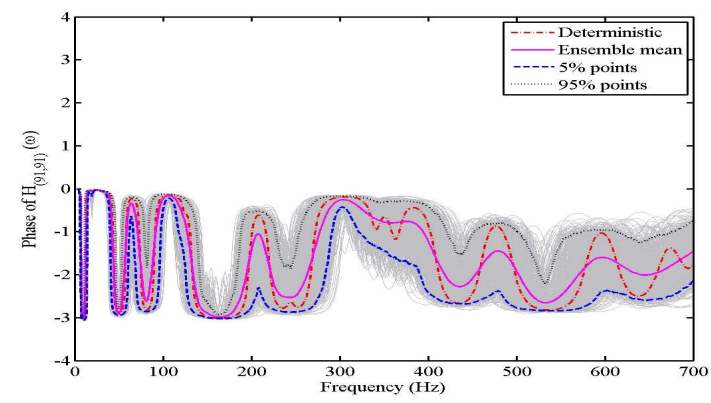

(b) Phase in the Low-frequency range

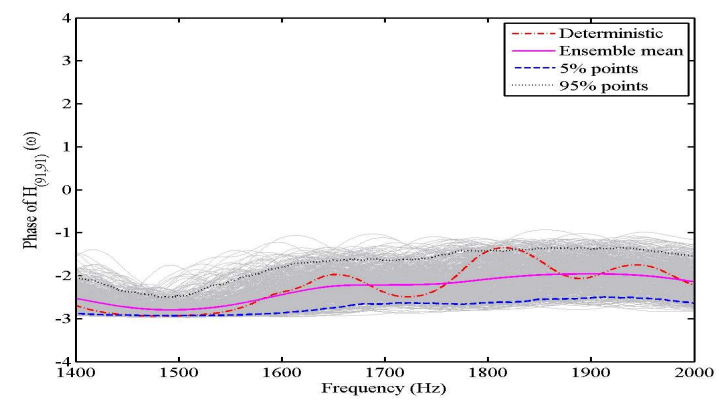

(d) Phase in the High-frequency range

Figure 6. Direct stochastic finite-element Monte Carlo Simulation of the phase of the driving-point-FRF of the plate with randomly distributed material properties.

element property functions are random fields, nature of these random fields (correlation structure, Gaussian or nonGaussian) and the amount of randomness are not used in the noncentral Wishart matrix approach. This is aimed at depicting a realistic situation where accurate information regarding these quantities are in general not available. Using $n=168, \delta_{M}=0.1194, \delta_{K}=0.2711$ and the covariance matrices, together with the deterministic values of $\mathbf{M}$ and $\mathbf{K}$, from the iterative procedure in subsection $\mathrm{C}$ we obtain

$$
p_{M}=15256 \text { and } \quad p_{K}=2713 \text {. }
$$

Using these, the samples of the mass and stiffness matrices are simulated following the procedure outlined in section V The amplitude and the phase of the cross-FRF obtained using the samples of the noncentral Wishart matrices are shown in Figs. 7 and 8 respectively. The realizations of the amplitude and phase of the FRF for each sample are shown together with the ensemble mean, $5 \%$ and $95 \%$ probability points and the results for the baseline system. In Figs. 7(b)-(d) and 8(b)-(d) the low, medium and high-frequency response, obtained by zooming in the appropriate frequency ranges in Figs. 7(a) and 8(a), are shown separately. The equivalent results for the driving-point-FRF are shown in Figs. 9 and 10 It may be observed that the qualitative features of the response is similar to what obtained using the detailed stochastic finite element simulation in Figs. $3-6$.

The predicted mean and standard deviation of the amplitude and phase using the direct stochastic finite element simulation and the noncentral Wishart matrix approach are compared in Figs. 11 - 14 for the cross-FRF and the drivingpoint-FRF respectively. In Figs. 11(b)-(d), 12(b)-(d), 13 (b)-(d) and 14(b)-(d) the low, medium and high-frequency response, obtained by zooming in the appropriate frequency ranges are shown respectively. The mean of the response amplitude obtained from the proposed noncentral Wishart matrix approach is very close to the results obtained using the stochastic finite element simulation across the frequency range considered. The standard deviations of the response amplitude obtained from the proposed noncentral Wishart matrix theory match reasonably well to the standard deviations obtained using the stochastic finite element simulation. It is interesting to note that the proposed noncentral Wishart matrix approach produces accurate results in the medium frequency range. For the low frequency region, the predication of the standard deviation is better around the resonance compared to the antiresonance. This is promising because the response around the resonance peaks are often most useful in practice.

The predicted $5 \%$ and $95 \%$ probability points using the direct stochastic finite element simulation and proposed

$$
14 \text { of } 22
$$




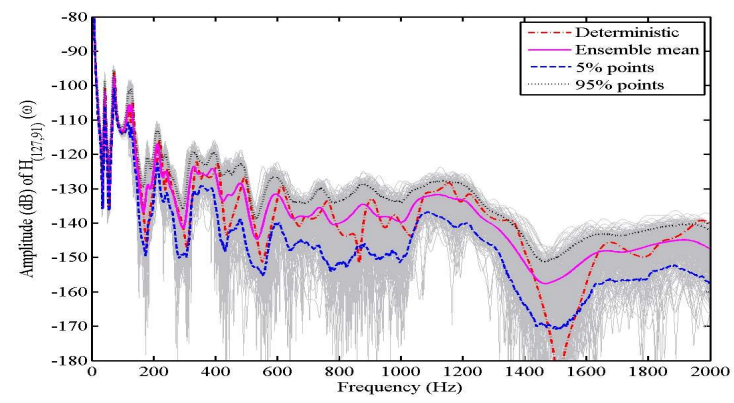

(a) Amplitude across the frequency range

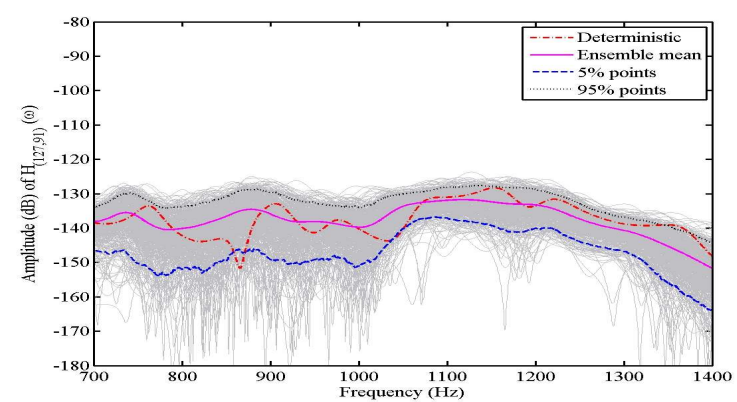

(c) Amplitude in the Medium-frequency range

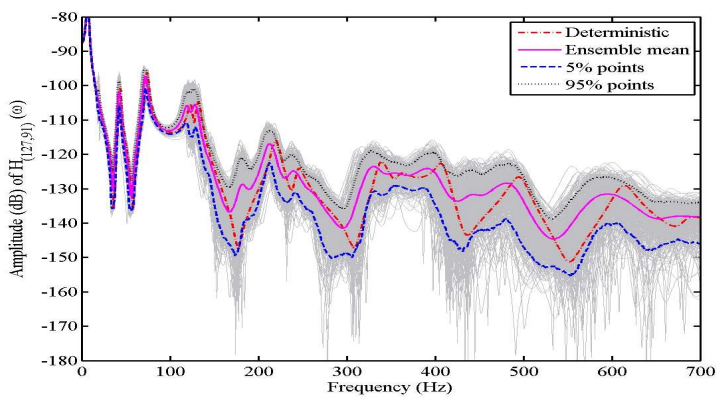

(b) Amplitude in the Low-frequency range

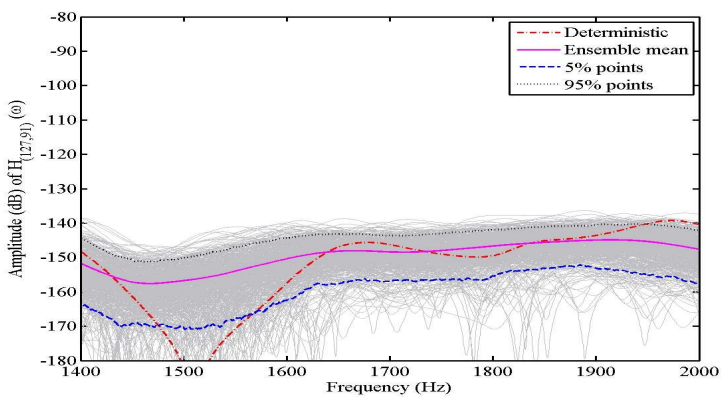

(d) Amplitude in the High-frequency range

Figure 7. Monte Carlo Simulation of the amplitude of the cross-FRF of the plate using noncentral Wishart mass and stiffness matrices, $n=168, \delta_{M}=0.1194$ and $\delta_{K}=0.2711$.

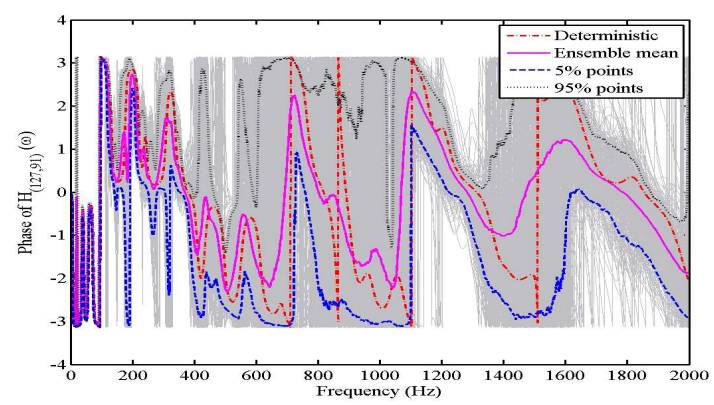

(a) Phase across the frequency range

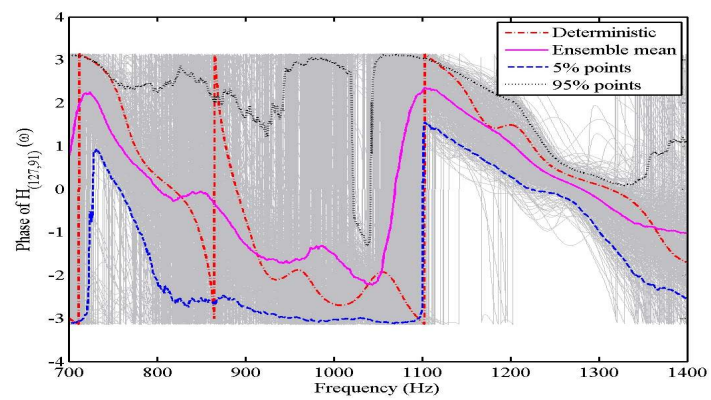

(c) Phase in the Medium-frequency range

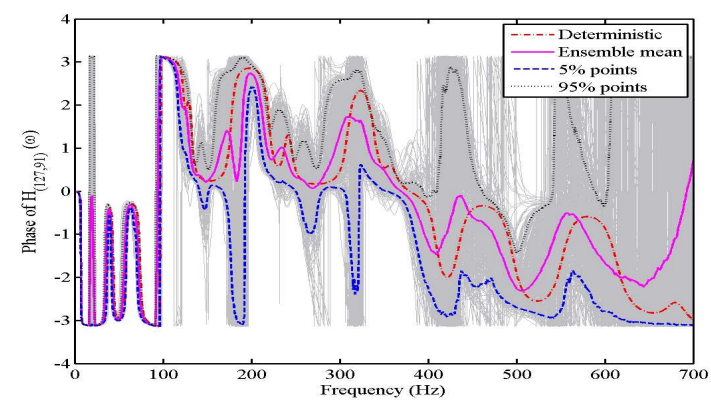

(b) Phase in the Low-frequency range

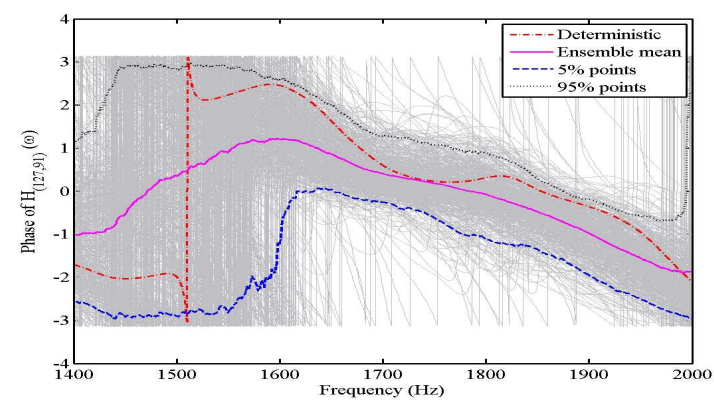

(d) Phase in the High-frequency range

Figure 8. Monte Carlo Simulation of the phase of the cross-FRF of the plate using noncentral Wishart mass and stiffness matrices, $n=168$, $\delta_{M}=0.1194$ and $\delta_{K}=0.2711$. 


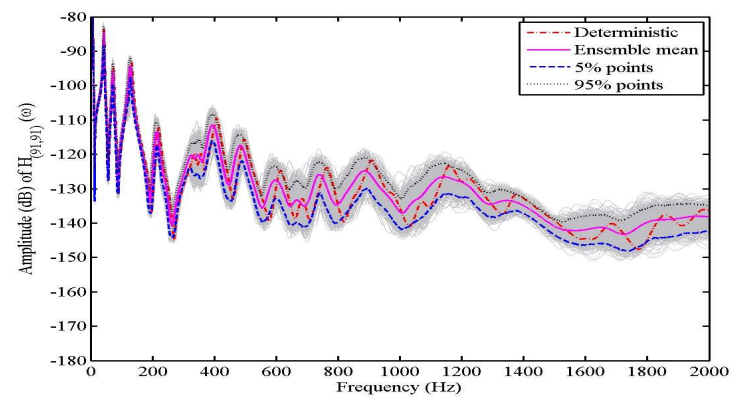

(a) Amplitude across the frequency range

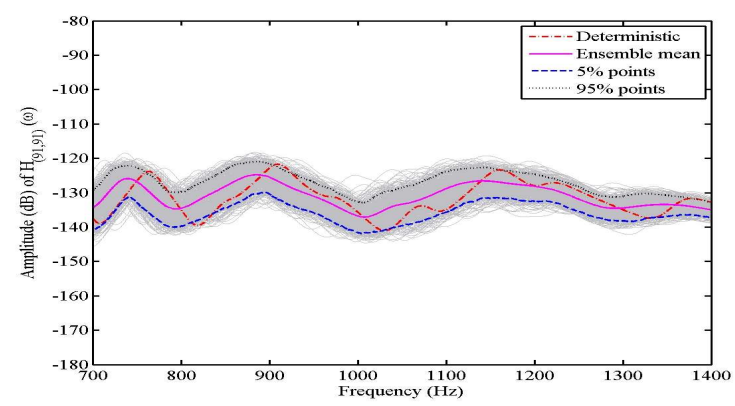

(c) Amplitude in the Medium-frequency range

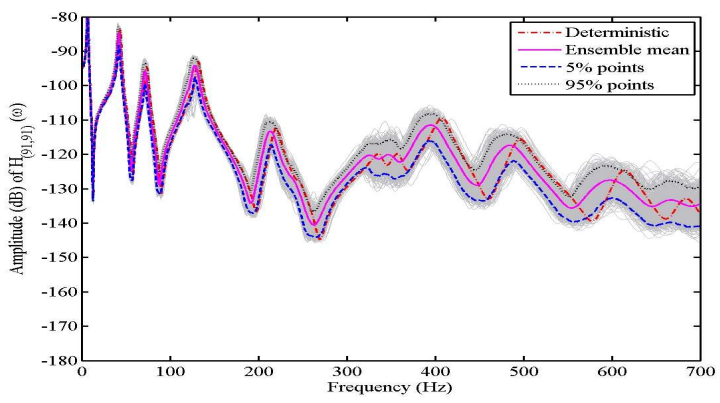

(b) Amplitude in the Low-frequency range

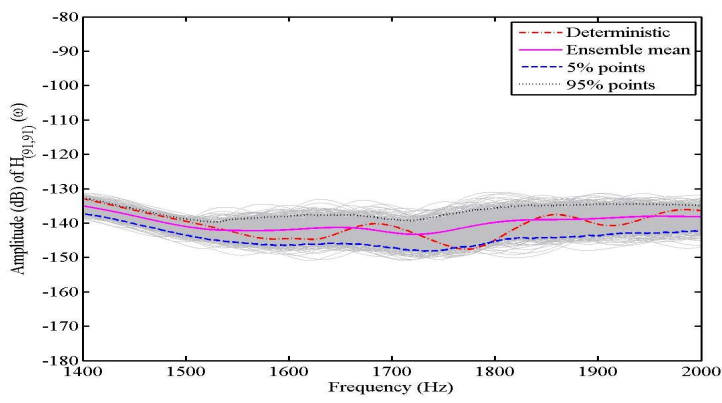

(d) Amplitude in the High-frequency range

Figure 9. Monte Carlo Simulation of the amplitude of the driving-point-FRF of the plate using noncentral Wishart mass and stiffness matrices, $n=168, \delta_{M}=0.1194$ and $\delta_{K}=0.2711$.

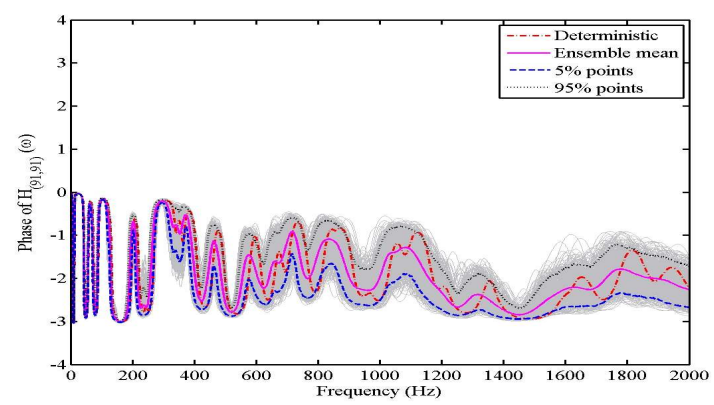

(a) Phase across the frequency range

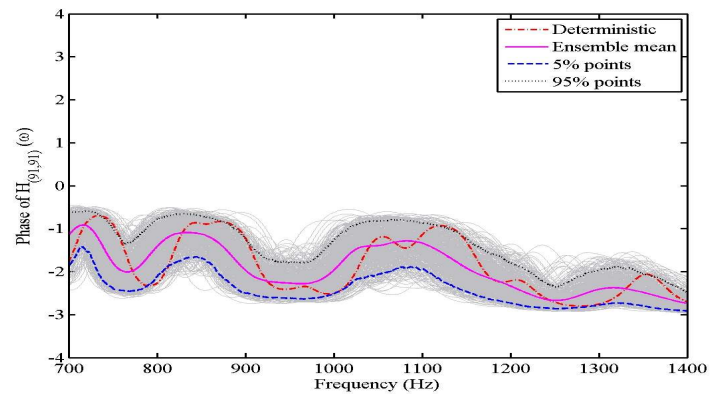

(c) Phase in the Medium-frequency range

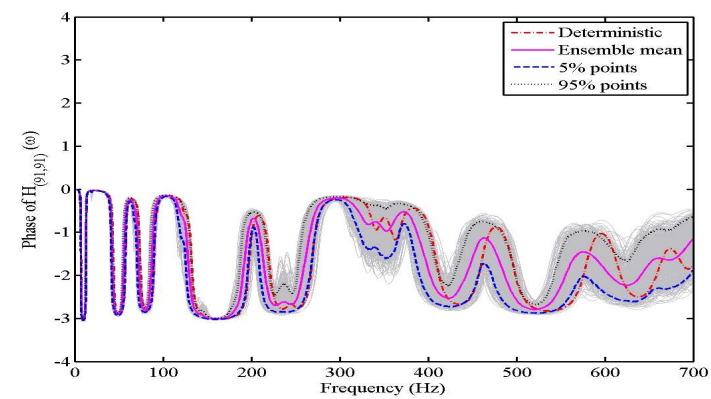

(b) Phase in the Low-frequency range

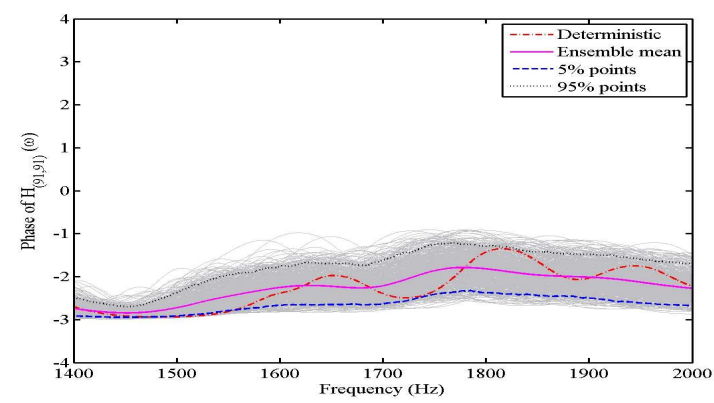

(d) Phase in the High-frequency range

Figure 10. Monte Carlo Simulation of the phase of the driving-point-FRF of the plate using noncentral Wishart mass and stiffness matrices, $n=168, \delta_{M}=0.1194$ and $\delta_{K}=0.2711$. 


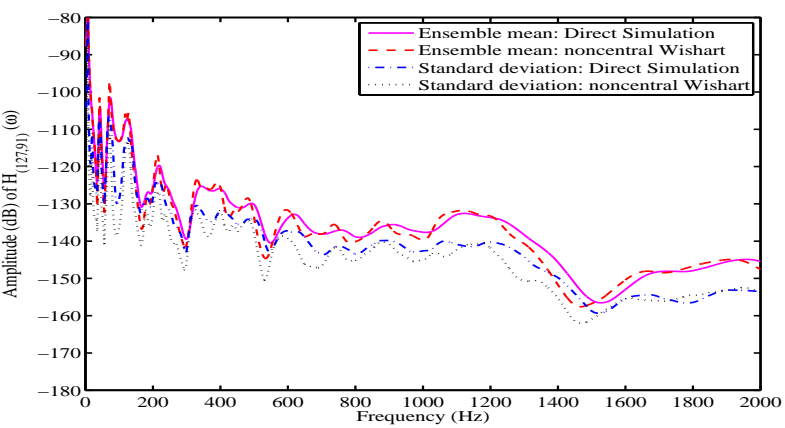

(a) Amplitude across the frequency range

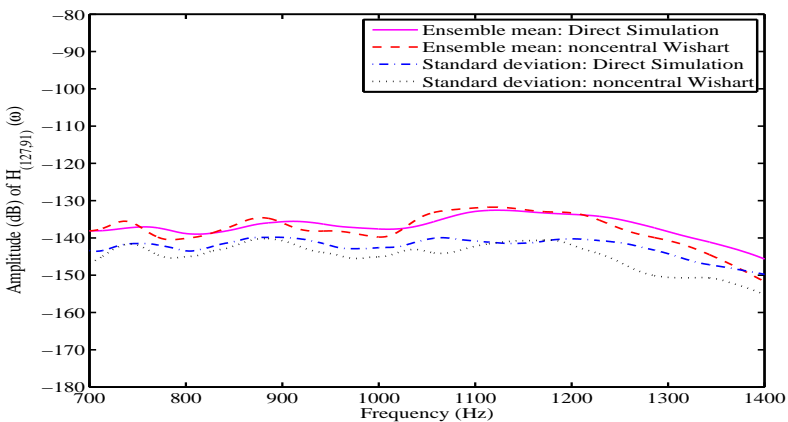

(c) Amplitude in the Medium-frequency range

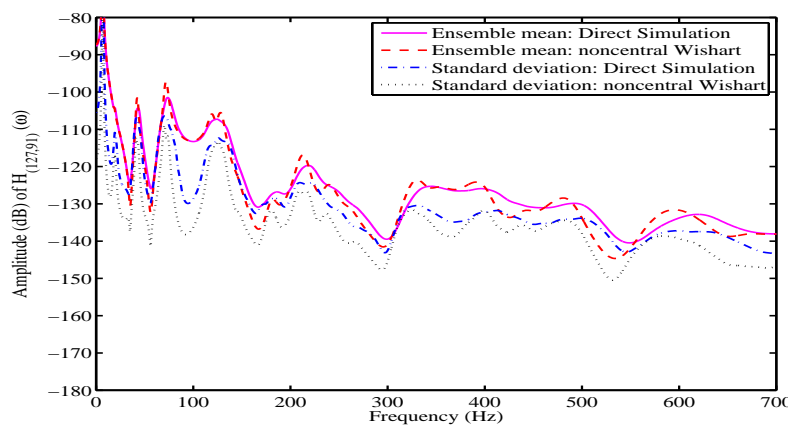

(b) Amplitude in the Low-frequency range

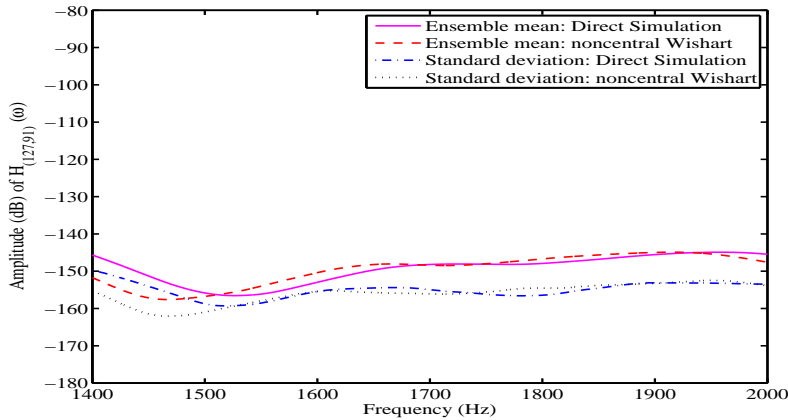

(d) Amplitude in the High-frequency range

Figure 11. Comparison of the mean and standard deviation of the amplitude of the cross-FRF obtained using the direct stochastic finite element simulation and proposed noncentral Wishart matrix method.

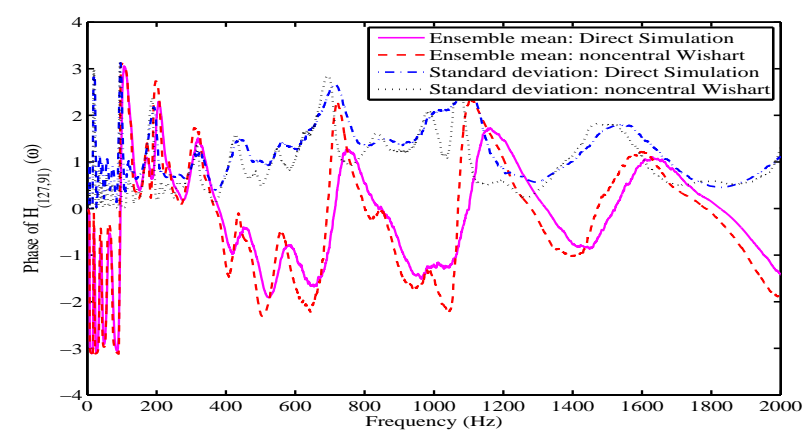

(a) Phase across the frequency range

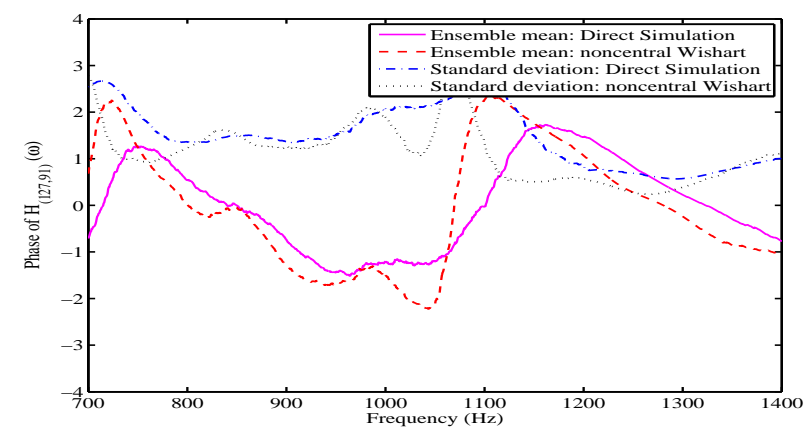

(c) Phase in the Medium-frequency range

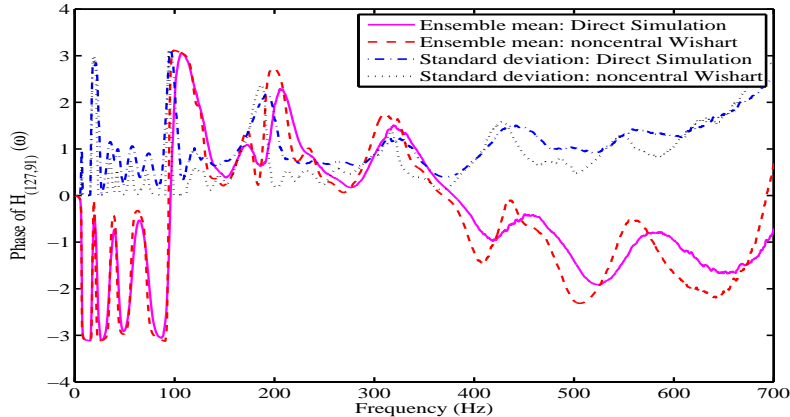

(b) Phase in the Low-frequency range

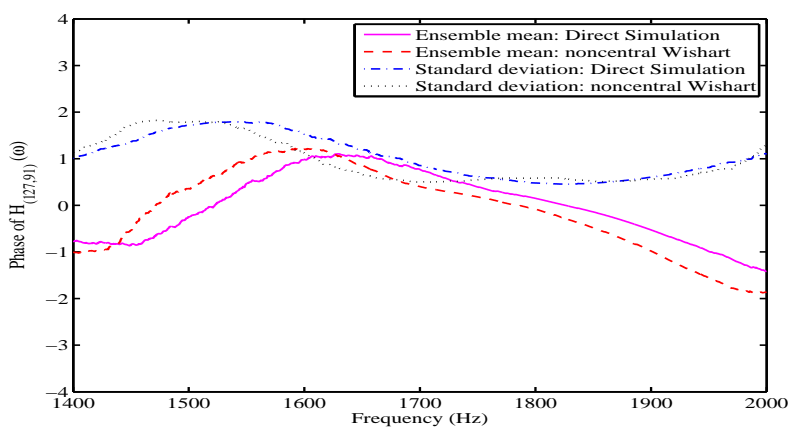

(d) Phase in the High-frequency range

Figure 12. Comparison of the mean and standard deviation of the phase of the cross-FRF obtained using the direct stochastic finite element simulation and proposed noncentral Wishart matrix method. 


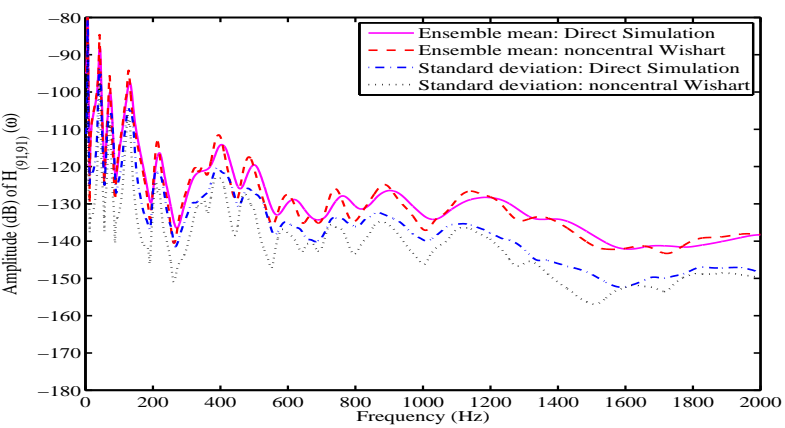

(a) Amplitude across the frequency range

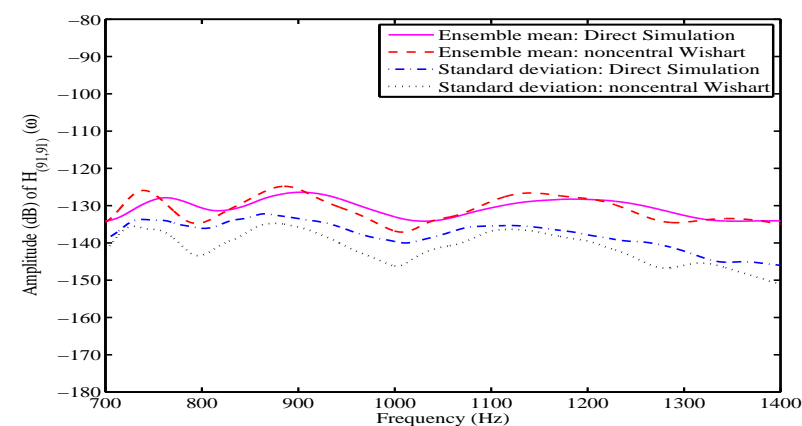

(c) Amplitude in the Medium-frequency range

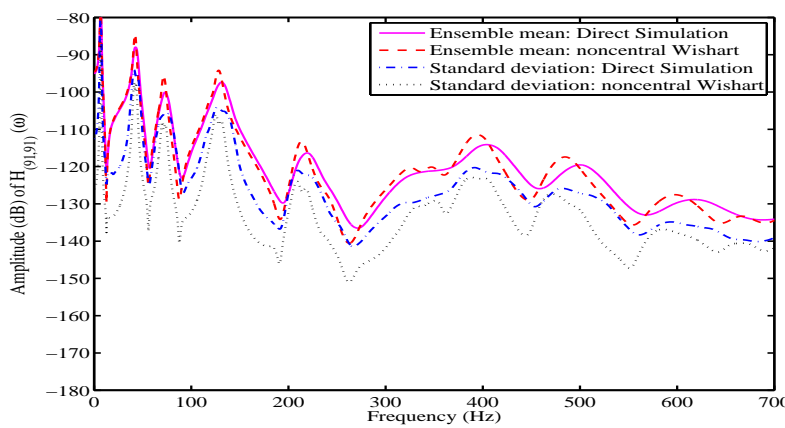

(b) Amplitude in the Low-frequency range

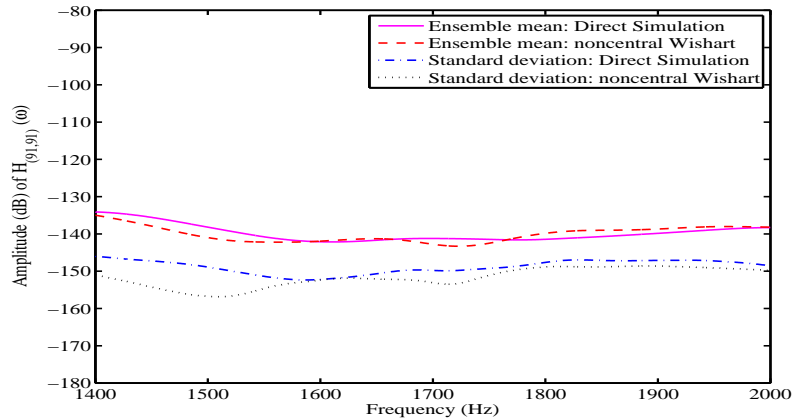

(d) Amplitude in the High-frequency range

Figure 13. Comparison of the mean and standard deviation of the amplitude of the driving-point-FRF obtained using the direct stochastic finite element simulation and proposed noncentral Wishart matrix method.

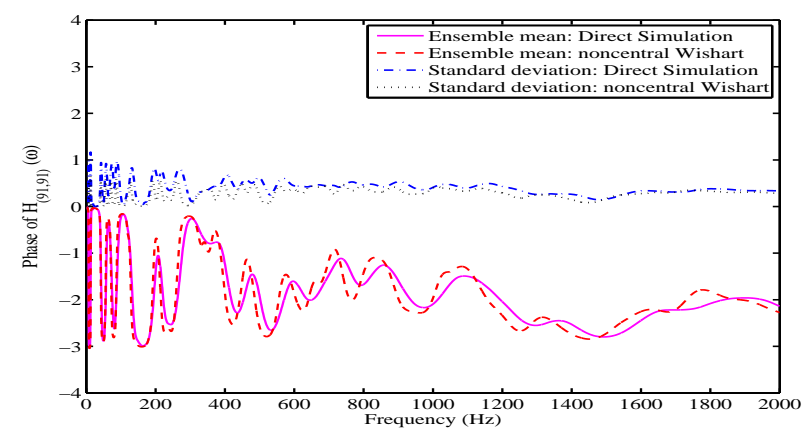

(a) Phase across the frequency range

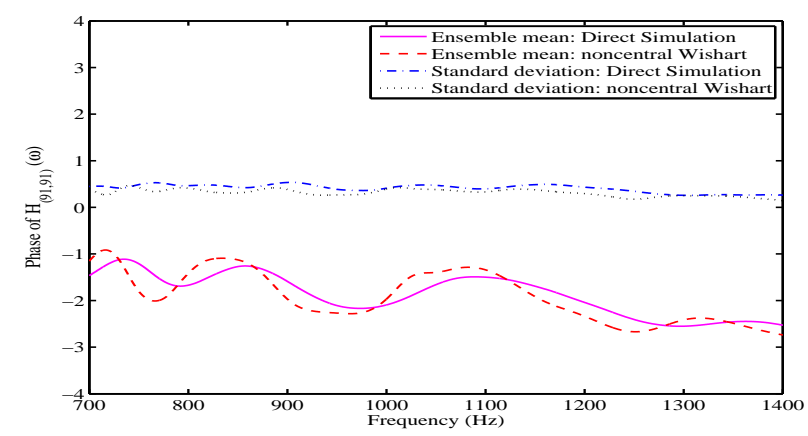

(c) Phase in the Medium-frequency range

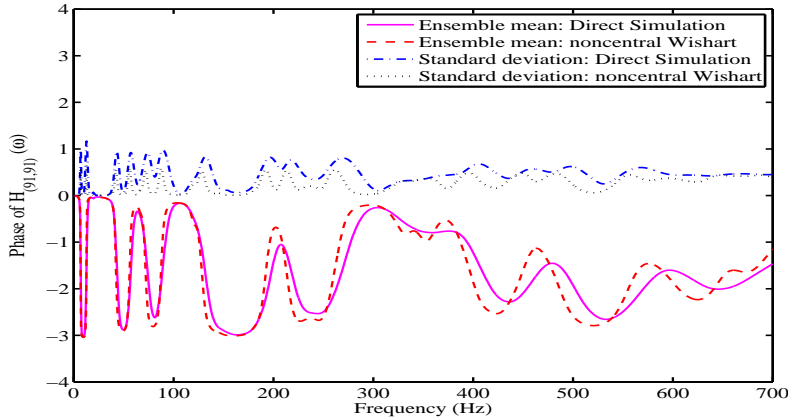

(b) Phase in the Low-frequency range

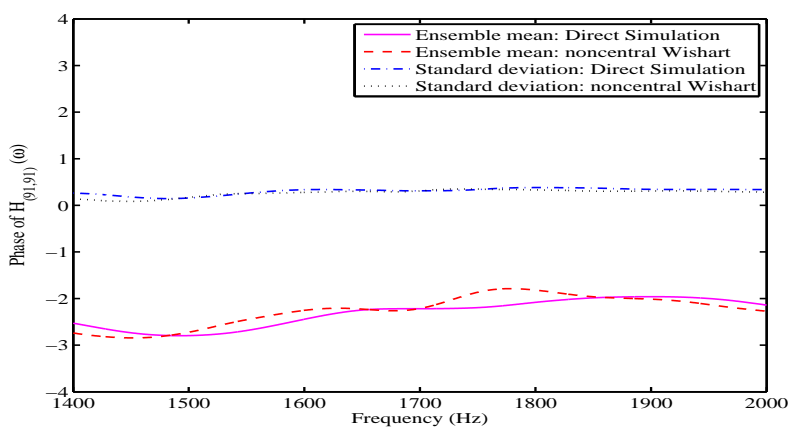

(d) Phase in the High-frequency range

Figure 14. Comparison of the mean and standard deviation of the phase of the driving-point-FRF obtained using the direct stochastic finite element simulation and proposed noncentral Wishart matrix method. 
noncentral Wishart matrix method are compared in Figs. 15-18. The essential feature of these plots are similar to the standard deviation plots shown before.

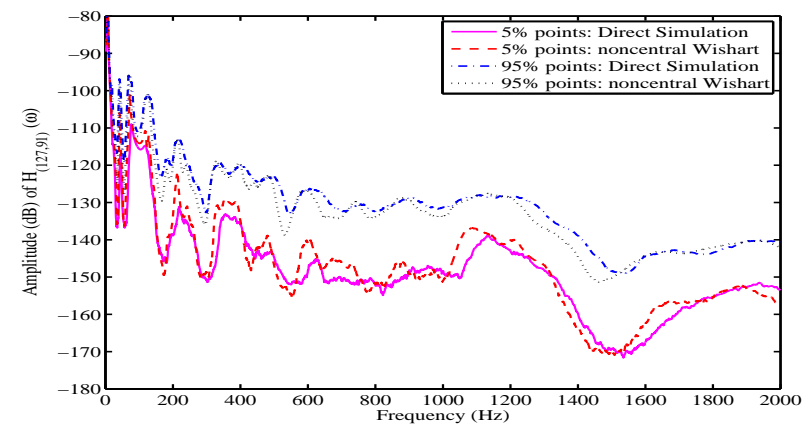

(a) Amplitude across the frequency range

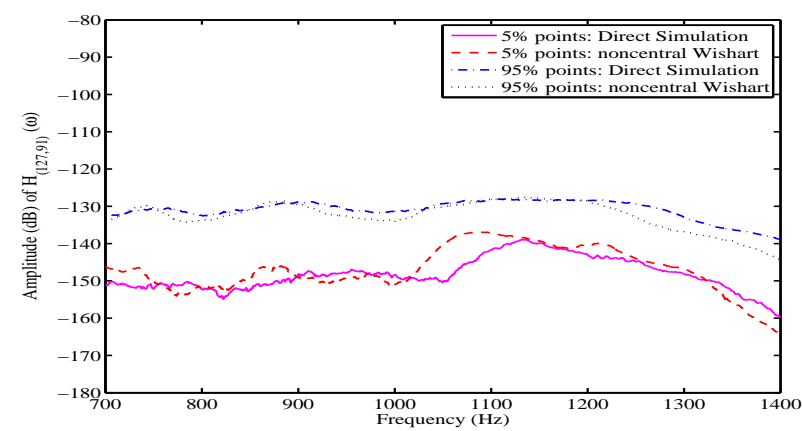

(c) Amplitude in the Medium-frequency range

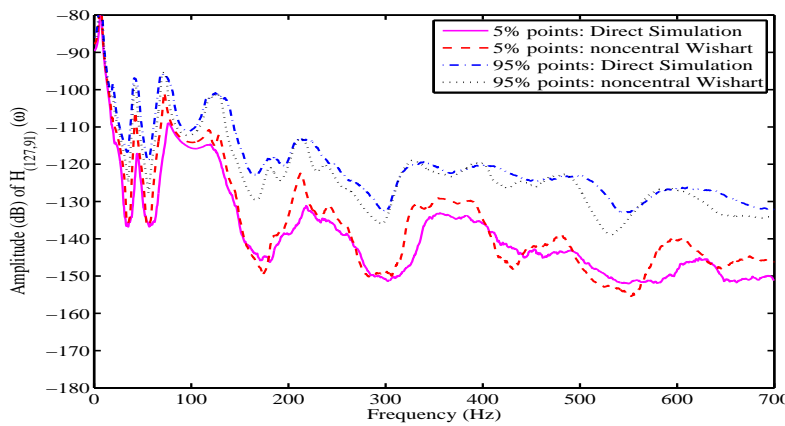

(b) Amplitude in the Low-frequency range

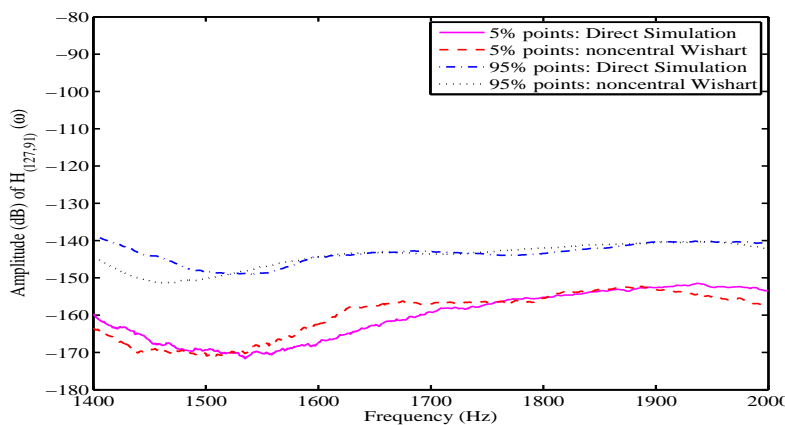

(d) Amplitude in the High-frequency range

Figure 15. Comparison of the $5 \%$ and $95 \%$ probability points of the amplitude of the cross-FRF obtained using the direct stochastic finite element simulation and proposed noncentral Wishart matrix method.

\section{Conclusions}

When uncertainties in the system parameters (parametric uncertainty) and modelling (nonparametric) are considered, the discretized equation of motion of linear dynamical systems is characterized by random mass, stiffness and damping matrices. A new unified parametric-nonparametric uncertainty quantification (UQ) method for linear dynamical systems has been proposed. Based on a matrix factorization approach, it is shown that the matrix variate probability density function of the random system matrices can be represented by the so called noncentral Wishart distribution. Existing nonparametric distribution is the central Wishart distribution, which is a special case of the proposed noncentral Wishart distribution. Matrix variate distributions proposed for structural dynamic problems are summarized in Table 1. The noncentral Wishart distribution has more parameters than the nonparametric central Wishart distribution to accommodate the parametric nature of uncertainty. The discovery of the noncentral Wishart distribution in this context is significant. On the one hand it eliminates some of the drawbacks of the nonparametric distribution, and in the other hand it can incorporate some parametric features while keeping the nonparametric features unchanged. This approach is particularly suitable when all elements of the covariance matrix of the system matrices are not known accurately (e.g, due to the lack of knowledge regarding the correlation functions, correlation lengths and standard deviations of the uncertain parameters).

A simple numerical simulation algorithm (which only requires the generation of Gaussian random numbers) has been outlined to implement the new distribution for structural dynamic problems. The derived noncentral Wishart random matrix model is applied to the forced vibration problem of a plate with stochastically inhomogeneous properties. Numerical results show that it is possible to predict the variation of the dynamic response using the noncentral Wishart matrices across a wide range of driving frequency. These results suggest that the noncentral Wishart matrices may be used as a consistent and unified uncertainty quantification tool valid across the whole frequency spectrum for linear vibration problems. 


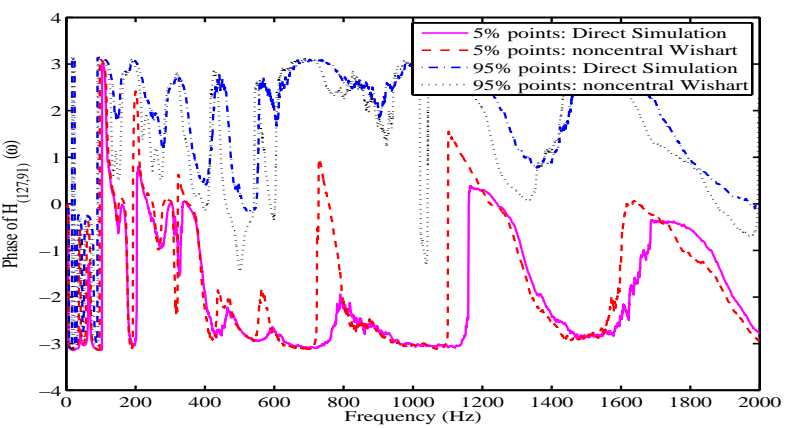

(a) Phase across the frequency range

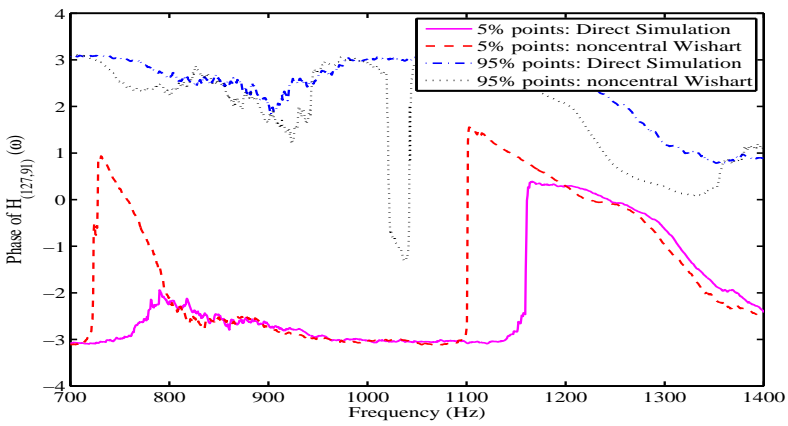

(c) Phase in the Medium-frequency range

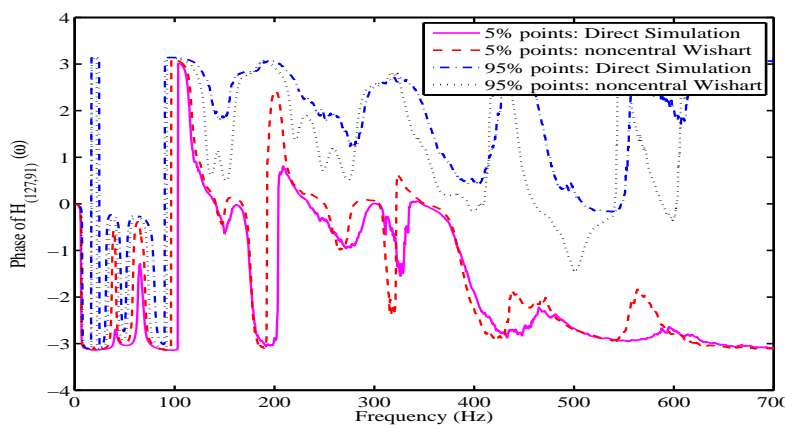

(b) Phase in the Low-frequency range

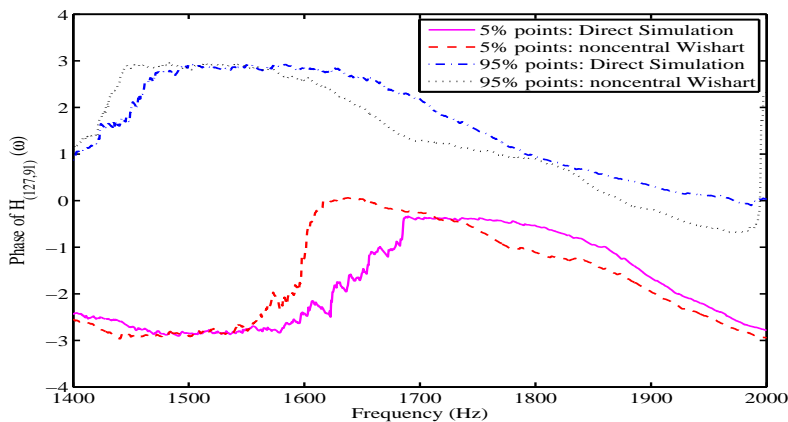

(d) Phase in the High-frequency range

Figure 16. Comparison of the $5 \%$ and $95 \%$ probability points of the phase of the cross-FRF obtained using the direct stochastic finite element simulation and proposed noncentral Wishart matrix method.

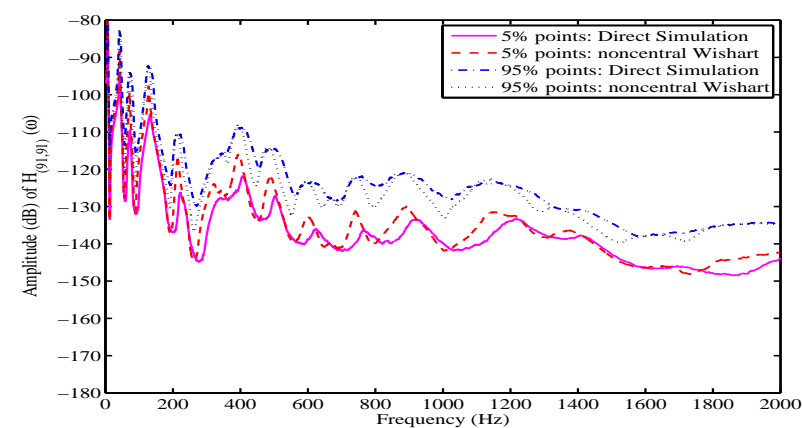

(a) Amplitude across the frequency range

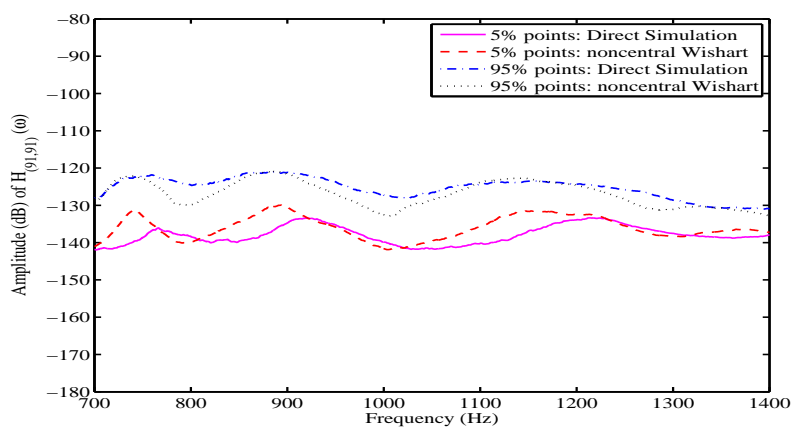

(c) Amplitude in the Medium-frequency range

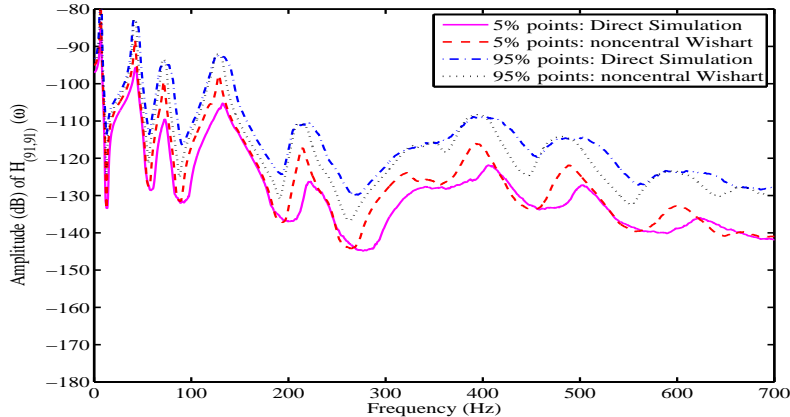

(b) Amplitude in the Low-frequency range

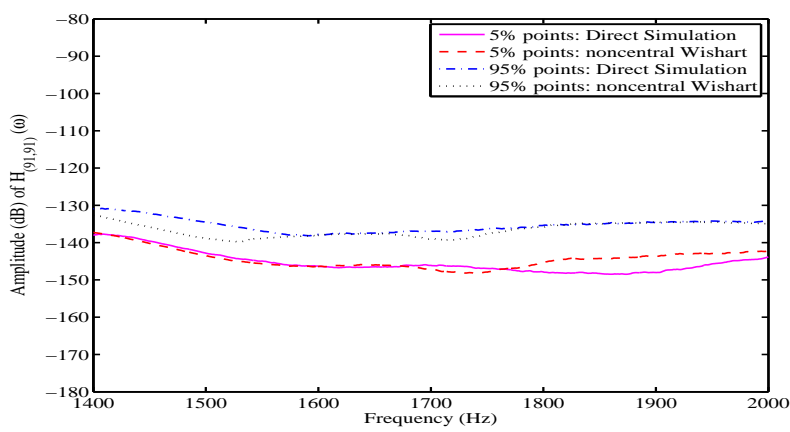

(d) Amplitude in the High-frequency range

Figure 17. Comparison of the 5\% and 95\% probability points of the amplitude of the driving-point-FRF obtained using the direct stochastic finite element simulation and proposed noncentral Wishart matrix method. 


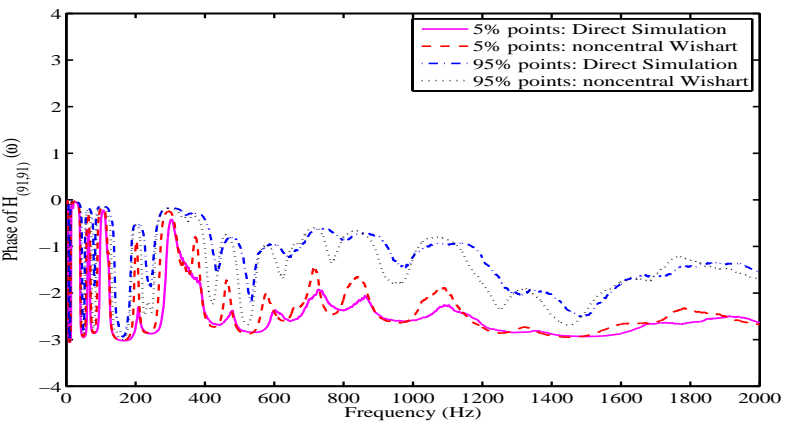

(a) Phase across the frequency range

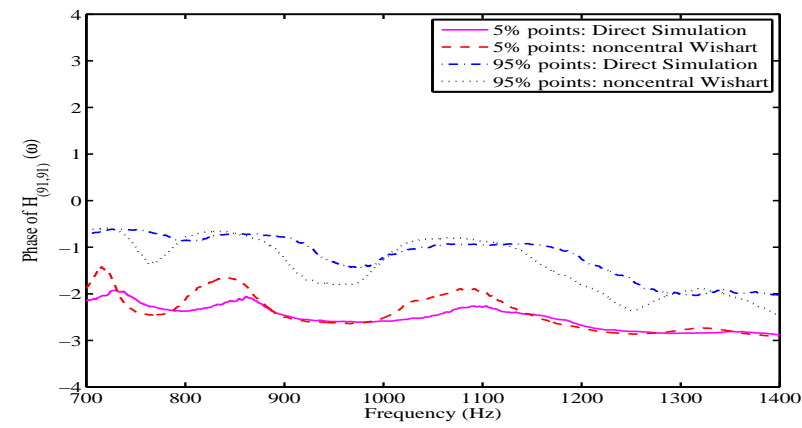

(c) Phase in the Medium-frequency range

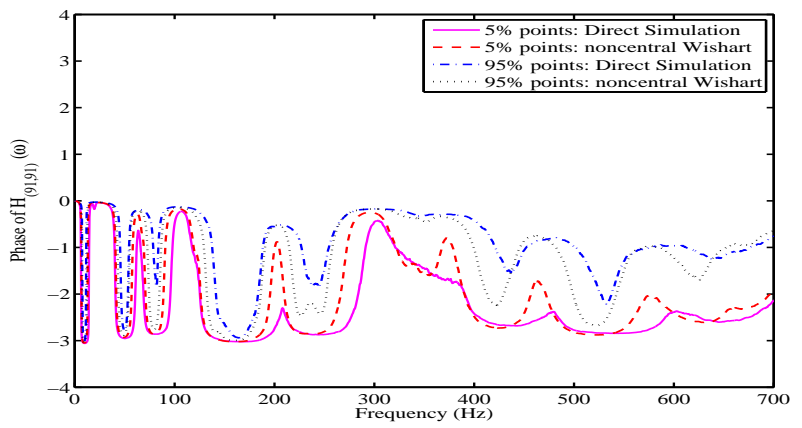

(b) Phase in the Low-frequency range

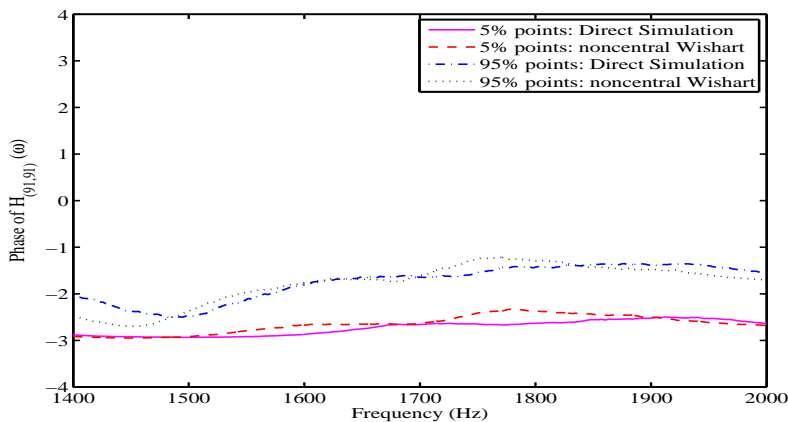

(d) Phase in the High-frequency range

Figure 18. Comparison of the $5 \%$ and $95 \%$ probability points of the phase of the driving-point-FRF obtained using the direct stochastic finite element simulation and proposed noncentral Wishart matrix method.

\section{Acknowledgments}

The author acknowledges the support of the UK Engineering and Physical Sciences Research Council (EPSRC) through the award of an Advanced Research Fellowship, grant number GR/T03369/01.

\section{References}

${ }^{1}$ Magnus, J. R. and Neudecker, H., Matrix Differential Calculus with Applications in Statistics and Econometrics, John Wiley \& Sons, New York, 1999.

${ }^{2}$ Harville, D. A., Matrix Algebra from a Statistician's Perspective, Springer-Verlag, New York, 1998.

${ }^{3}$ Graham, A., Kronecker Products and Matrix Calculus With Applications, Mathematics and its Applications, Ellis Horwood Limited, Chichester, UK, 1981

${ }^{4}$ Hemez, F. M., "Overview of Uncertainty Quantification and Model Verification and Validation" Pan American Advanced Study Institute (PASI) on Damage Prognosis, Florianopolis, Brazil, October 19-31 2003.

${ }^{5}$ Hemez, F. M., 'Uncertainty Quantification and the Verification and Validation of Computational Models" Damage Prognosis for Aerospace, Civil and Mechanical Systems, edited by D. J. Inman, C. R. Farrar, V. L. Jr., , and V. S. Jr., John Wiley \& Sons Ltd., London, United Kingdom, December 2004.

${ }^{6}$ Shinozuka, M. and Yamazaki, F., "Stochastic finite element analysis: an introduction," Stochastic structural dynamics: Progress in theory and applications, edited by S. T. Ariaratnam, G. I. Schueller, and I. Elishakoff, Elsevier Applied Science, London, 1998

${ }^{7}$ Ghanem, R. and Spanos, P., Stochastic Finite Elements: A Spectral Approach, Springer-Verlag, New York, USA, 1991

${ }^{8}$ Kleiber, M. and Hien, T. D., The Stochastic Finite Element Method, John Wiley, Chichester, 1992.

${ }^{9}$ Matthies, H. G., Brenner, C. E., Bucher, C. G., and Soares, C. G., "Uncertainties in probabilistic numerical analysis of structures and solids - Stochastic finite elements," Structural Safety, Vol. 19, No. 3, 1997, pp. 283-336.

${ }^{10}$ Manohar, C. S. and Adhikari, S., "Dynamic stiffness of randomly parametered beams," Probabilistic Engineering Mechanics, Vol. 13, No. 1, January 1998, pp. 39-51.

${ }^{11}$ Adhikari, S. and Manohar, C. S., "Dynamic analysis of framed structures with statistical uncertainties," International Journal for Numerical Methods in Engineering, Vol. 44, No. 8, 1999, pp. 1157-1178.

${ }^{12}$ Adhikari, S. and Manohar, C. S., "Transient dynamics of stochastically parametered beams," ASCE Journal of Engineering Mechanics, Vol. 126, No. 11, November 2000, pp. 1131-1140.

${ }^{13}$ Haldar, A. and Mahadevan, S., Reliability Assessment Using Stochastic Finite Element Analysis, John Wiley and Sons, New York, USA, 2000.

${ }^{14}$ Sudret, B. and Der-Kiureghian, A., "Stochastic Finite Element Methods and Reliability," Tech. Rep. UCB/SEMM-2000/08, Department of Civil \& Environmental Engineering, University Of California, Berkeley, November 2000

${ }^{15}$ Elishakoff, I. and Ren, Y. J., Large Variation Finite Element Method for Stochastic Problems, Oxford University Press, Oxford, U.K., 2003.

${ }^{16}$ Adhikari, S., "On the quantification of damping model uncertainty," Journal of Sound and Vibration, 2007, to appear. 
Table 1. Hierarchy of Random Matrix $(\mathbf{R M})$ models for linear uncertain dynamical systems. Here $\mathbf{G} \equiv\{\mathbf{M}, \mathbf{C}, \mathbf{K}\}$ is any system matrix, $\overline{\mathbf{G}} \in \mathbb{R}_{n}^{+}$is the baseline (mean) model and $\mathbf{C}_{G}=\operatorname{cov}(\operatorname{vec}(\mathbf{G})) \in \mathbb{R}_{n^{2}}^{+}$is the covariance matrix of the elements of $\mathbf{G}$.

\begin{tabular}{|c|c|c|}
\hline Author/Year & Random Matrix Model & Comments \\
\hline $\begin{array}{l}\text { Soize }^{17}(2005) \text { [and } \\
\text { references therein] }\end{array}$ & $\begin{array}{l}\text { Central Wishart/gamma random matrix } \\
W_{n}(p, \boldsymbol{\Sigma}) \text {, with } \boldsymbol{\Sigma}=\overline{\mathbf{G}} / p \\
p=\frac{1}{\delta_{G}^{2}}\left\{1+\frac{\{\operatorname{Trace}(\overline{\mathbf{G}})\}^{2}}{\operatorname{Trace}\left(\overline{\mathbf{G}}^{2}\right)}\right\} \text { and } \\
\delta_{G}^{2}=\frac{\mathrm{E}\left[\|\mathbf{G}-\mathrm{E}[\mathbf{G}]\|_{\mathrm{F}}^{2}\right]}{\|\mathrm{E}[\mathbf{G}]\|_{\mathrm{F}}^{2}}=\frac{\operatorname{Trace}\left(\mathbf{C}_{G}\right)}{\operatorname{Trace}\left(\overline{\mathbf{G}}^{2}\right)}\end{array}$ & $\begin{array}{l}\text { (a) The trace of the covariance matrix of } \\
\text { the elements of a system matrix is required. } \\
\text { (b) The mean of the inverse and the inverse } \\
\text { of the mean of the system matrices can be } \\
\text { significantly different from each other for } \\
\text { the choice of the distribution parameters. }\end{array}$ \\
\hline Adhikari $^{18,19}$ (2006) & $\begin{array}{l}\text { Central Wishart/gamma random matrix } \\
W_{n}(p, \boldsymbol{\Sigma}) \text {, with } \boldsymbol{\Sigma}=\overline{\mathbf{G}} / \sqrt{p(p-n-1)} \\
\text { and the rest is as defined above. }\end{array}$ & $\begin{array}{l}\text { (a) The trace of the covariance matrix of } \\
\text { the elements of a system matrix is required. } \\
\text { (b) Parameters are obtained using a least- } \\
\text { square error minimization approach. The } \\
\text { mean of the matrix and its inverse produce } \\
\text { minimum deviations from their respective } \\
\text { deterministic values. }\end{array}$ \\
\hline
\end{tabular}

This paper Noncentral Wishart random matrix

$W_{n}(p, \boldsymbol{\Sigma}, \boldsymbol{\Theta})$, with
$\boldsymbol{\Sigma}=(\overline{\mathbf{G}}-\boldsymbol{\Omega}) / p, \boldsymbol{\Theta}=\boldsymbol{\Sigma}^{-1} \boldsymbol{\Omega}, p=$
$\frac{\operatorname{Trace}\left(\overline{\mathbf{G}}^{2}-\boldsymbol{\Omega}^{2}\right)+\{\operatorname{Trace}(\overline{\mathbf{G}})\}^{2}-\{\operatorname{Trace}(\boldsymbol{\Omega})\}^{2}}{\delta_{G}^{2} \operatorname{Trace}\left(\overline{\mathbf{G}}^{2}\right)}$
$\boldsymbol{\Omega} \otimes \boldsymbol{\Omega}=\overline{\mathbf{G}} \otimes \overline{\mathbf{G}}-p \mathbf{C}_{G} / 2$ and $\delta_{G}$ is as defined above.

(a) This distribution requires the same information as the previous two distributions, namely $\overline{\mathbf{G}} \in \mathbb{R}_{n}^{+}$and $\mathbf{C}_{G} \in \mathbb{R}_{n^{2}}^{+}$.

(b) If $\boldsymbol{\Omega}=\mathbf{O}_{n, n}$ then this distribution reduces to the central distribution proposed before.

(c) The matrix $\Omega \in \mathbb{R}_{n}^{+}$captures the parametric uncertainty through a least-square error minimization involving the covariance matrix $\mathbf{C}_{G}$.

\footnotetext{
${ }^{17}$ Soize, C., "Random matrix theory for modeling uncertainties in computational mechanics," Computer Methods in Applied Mechanics and Engineering, Vol. 194, No. 12-16, 2005, pp. 1333-1366.

${ }^{18}$ Adhikari, S., "A non-parametric approach for uncertainty quantification in elastodynamics," 47th AIAA/ASME/ASCE/AHS/ASC Structures, Structural Dynamics \& Materials Conference, Newport, Rhode Island, USA, May 2006.

${ }^{19}$ Adhikari, S., "Matrix variate distributions for probabilistic structural mechanics," AIAA Journal, 2007, accepted for publication.

${ }^{20}$ Adhikari, S., Lonkar, K., and Friswell, M. I., "Experimental case studies on uncertainty quantification in structural dynamics," Proceedings of the 25th International Modal Analysis Conference (IMAC-XXV), Orlando, Florida, USA, February 2007.

${ }^{21}$ Adhikari, S., Friswell, M. I., and Lonkar, K., "Uncertainty in structural dynamics: Experimental case studies on beams and plates," Proceedings of the Computational Methods in Structural Dynamics and Earthquake Engineering (COMPDYN 2007), Crete, Greece, June 2007.

${ }^{22}$ Wishart, J., "The generalized product moment distribution in samples from a normal multivariate population," Biometrika, Vol. 20, No. A, 1928 , pp. 32-52.

${ }^{23}$ Mezzadri, F. and Snaith, N. C., editors, Recent Perspectives in Random Matrix Theory and Number Theory, London Mathematical Society Lecture Note, Cambridge, U. K., 2005, Cambridge University Press.

${ }^{24}$ Tulino, A. M. and Verdú, S., Random Matrix Theory and Wireless Communications, now Publishers Inc., Hanover, MA, USA, 2004.

${ }^{25}$ Eaton, M. L., Multivariate Statistics: A Vector Space Approach, John Wiley \& Sons, New York, 1983.

${ }^{26}$ Girko, V. L., Theory of Random Determinants, Kluwer Academic Publishers, The Neatherlands, 1990.

${ }^{27}$ Muirhead, R. J., Aspects of Multivariate Statistical Theory, John Wiely and Sons, New York, USA, 1982.

${ }^{28}$ Mehta, M. L., Random Matrices, Academic Press, San Diego, CA, 2nd ed., 1991.

${ }^{29}$ Gupta, A. and Nagar, D., Matrix Variate Distributions, Monographs \& Surveys in Pure \& Applied Mathematics, Chapman \& Hall/CRC, London, 2000

${ }^{30}$ Van Loan, C. F. and Pitsianis, N., "Approximation with Kronecker Products," Linear Algebra for Large Scale and Real-Time Applications, edited by M. S. Moonen and G. H. Golub, Kluwer Publications, The Netherlands, 1993, pp. 293-314.

${ }^{31}$ Langville, A. N. and Stewart, W. J., "The Kronecker product and stochastic automata networks," Journal of Computational and Applied Mathematics, Vol. 167, No. 2, 2004, pp. 429-447.

${ }^{32}$ Langville, A. N. and Stewart, W. J., "Kronecker product approximate preconditioner for SANs," Numerical Linear Algebra with Applications, Vol. 11, No. 8-9, 2004, pp. 723-752.

${ }^{33}$ Langville, A. N. and Stewart, W. J., "Testing the nearest Kronecker product preconditioner on Markov chains and stochastic automata networks," Informs Journal on Computing, Vol. 16, No. 3, 2004, pp. 300-315.
} 